D.E. Moulton

J. Lega 

We explore the effect of disjoining pressure on a thin film equation in the presence of a non-uniform body force, motivated by a model describing the reverse draining of a magnetic film. To this end, we use a combination of numerical investigations and analytical considerations. The disjoining pressure has a regularizing influence on the evolution of the system and appears to select a single steady-state solution for fixed height boundary conditions; this is in contrast with the existence of a continuum of locally attracting solutions that exist in the absence of disjoining pressure for the same boundary conditions. We numerically implement matched asymptotics expansions to construct equilibrium solutions and also investigate how they behave as the disjoining pressure is sent to zero. Finally, we consider the effect of the competition between forcing and disjoining pressure on the coarsening dynamics of the thin film for fixed contact angle boundary conditions. 


\title{
Effect of disjoining pressure in a thin film equation with non-uniform forcing
}

\author{
D.E. Moulton $†$ and J. Legał
}

(Received 24 August 2012)

\section{Introduction}

In this paper we consider thin film evolution equations on a finite domain with joint contributions from a disjoining pressure and a spatially dependent body force whose gradients are of order one. Our motivation comes from the study of ferrofluids. In the presence of a non-uniform magnetic field, the magnetic particles in a ferrofluid experience a force, a phenomenon known as magnetophoresis [15]. In [21], experiments were conducted with a draining magnetic soap film in the presence of a strong magnet. Using the setup pictured in Fig. 1, Moulton and Pelesko derived the following dimensionless

$\dagger$ Corresponding Author; OCCAM, Mathematical Institute, University of Oxford, UK;

University of Arizona, Department of Mathematics, Tucson, AZ (moulton@maths.ox.ac.uk)

$\ddagger$ University of Arizona, Department of Mathematics, Tucson, AZ (lega@math.arizona.edu) 


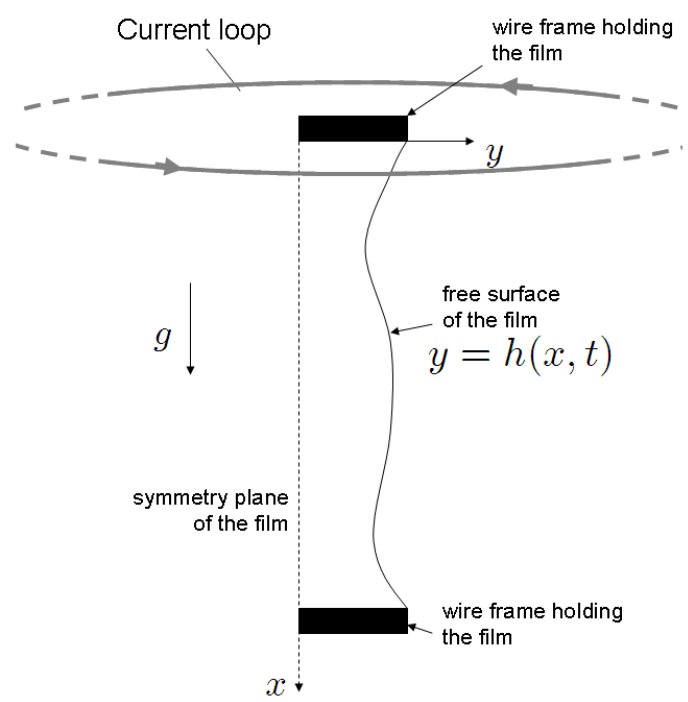

Figure 1. Setup for the draining magnetic film system of Refs. [21, 20]. [Reproduced from

$$
\text { [20], with permission] }
$$

evolution equation for the thickness of the draining vertical film

$$
h_{t}+\frac{\partial}{\partial x}\left(\frac{h^{3}}{3}\left(\sigma h_{x x x}+\gamma(x)\right)\right)=0 \text {, }
$$

where

$$
\gamma(x)=1-\frac{3 \lambda x}{\left(1+x^{2}\right)^{4}}
$$

Here $h(x, t)$ is the film half-thickness, $\sigma$ is an inverse capillary number characterising surface tension, and $\gamma(x)$ represents the spatially dependent body force: the first term corresponds to gravity, which acts in the positive $x$-direction, and the second term is the magnetic force acting along the axis of a current loop and pulling the film upward, i.e. in the negative $x$-direction. The constant $\lambda$ appears as a dimensionless parameter relating the magnetic force to gravity (for more details, see [21]). Figure 2 shows $\gamma$ and its antiderivative $\Gamma(x)$ as functions of $x$ for $\lambda=1$. 


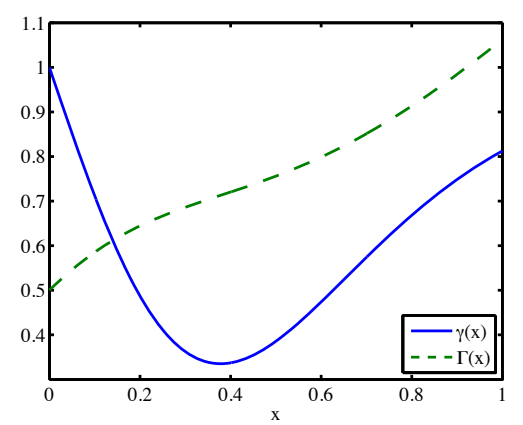

FiguRE 2. The forcing $\gamma(x)$ and its antiderivative $\Gamma(x)=x+\lambda /\left(2\left(1+x^{2}\right)^{3}\right)$, plotted for $\lambda=1$.

The dimensionless Equation (1.1) is reached through the standard lubrication approximation, with vertical lengths scaled by the length of the frame $L$, and the film halfthickness scaled by the height of the film on the frame $h_{0} .{ }^{1}$ Then $\epsilon=h_{0} / L$ is a small parameter. The vertical velocity component is scaled by $U_{0}=\rho g h_{0}^{2} / \mu$, where $g$ is gravity, $\rho$ is fluid density, and $\mu$ is dynamic viscosity; the horizontal velocity component is scaled by $\epsilon U_{0}$; and the time scale is $L / U_{0}$. In the experiments of [21], $L=5 \mathrm{~cm}, h_{0} \approx 50 \mu \mathrm{m}$ and the time scale is on the order of $5 \mathrm{~s}$. The magnetic parameter $\lambda$ ranges from 1 to 2 , which corresponds to a magnetic field strength on the order of $1500 \mathrm{G}$, and $\sigma$ ranges from 0.001 to 0.005 .

For strong enough magnetic field, $\gamma(x)$ can be made negative in portions of the domain, leading to a non-uniform body force in the vertical direction. In the experiments in [21], this was exploited to demonstrate the interesting phenomenon of reverse draining, in which the vertical force of the magnetic field overcame the downward gravitational force, and the soap film could be made to drain upward against gravity. Equilibrium solutions

1 We assume $h_{0}$ is a characteristic length in the horizontal direction, and thus use the same scaling for fixed contact angle boundary conditions, (BC2) discussed below. 
of the system (1.1), with fixed height boundary conditions and the forcing function $\gamma(x)$ either given by (1.2) or arbitrary, were analyzed in depth in [20]. The equilibrium solution set was shown to have a rich structure, with a strong connection between the form of the forcing function and the structure of equilibrium solutions. In particular, families of piecewise continuous equilibrium solutions were found, with $h=0$ over a portion of the domain and discontinuities in $h_{x x}$ at the piecewise junctions. Numerical simulations suggested convergence of the dynamics towards these piecewise solutions as time $t \rightarrow \infty$. In other words, the solutions continued to remain smooth as far as the numerics could be pushed and divergence of the third derivative seemed to only occur in infinite time. Also, based on numerical simulations, the continuum family of equilibrium solutions appeared to be locally attracting. However, since the limit $h \rightarrow 0$ represents rupture of the film, these solutions are not physically valid as equilibrium profiles, and it is expected that other effects, neglected in $[21,20]$, should come into play as the thickness of the film is reduced.

In particular, an important effect that was not included in $[21,20]$ is that of disjoining pressure (DP), which is used to account for intermolecular forces in very thin film regions. Letting $\Pi(h)$ denote the DP, the evolution equation (1.1) becomes

$$
h_{t}+\frac{\partial}{\partial x}\left(\frac{h^{3}}{3}\left(\sigma h_{x x x}+\Pi_{x}(h)+\gamma(x)\right)\right)=0 .
$$

Following [29] we consider the following form for DP

$$
\Pi(h)=A\left(\left(\frac{h^{*}}{h}\right)^{n}-\left(\frac{h^{*}}{h}\right)^{m}\right), n>m>1,
$$

where $A$ is a dimensionless pressure term (in [21] pressure is scaled by $\mu L U_{0} / h_{0}^{2}$ ). Asso- 
ciated with (1.4) is a disjoining energy

$$
\mathcal{E}_{\mathcal{D P}}(h)=-\int_{h^{*}}^{h} \Pi(\tilde{h}) d \tilde{h}
$$

which has a single stable minimum at the generally very thin film thickness $h=h^{*}$. This leads to an attractive force when $h>h^{*}$ and a repulsive force for $h<h^{*}$ that diverges as $h \rightarrow 0$. In terms of soap films, the DP term can be used to capture the presence of "black film": a thinning film that reaches a very small thickness, typically on the order of 100 $\mathrm{nm}$, at which point repulsion between the opposing surfaces of the film resists further thinning. The exact shape of the energy well depends on the exponents $n$ and $m$, and the strength of the DP, captured by the constant $A$.

The form of boundary conditions can be very important for film evolution and solution properties. Here, we study (1.3) on a finite length domain $x \in[0,1]$, and consider two types of boundary conditions at $x=0,1$. To investigate the regularizing role of DP, we use a fixed height boundary condition, which we denote (BC1). This is written

$$
(\mathrm{BC} 1): h(0)=h(1)=1 \text {. }
$$

To investigate the competition between forcing and disjoining pressure, we consider a fixed contact angle condition, denoted (BC2), which reads

$$
(\mathrm{BC} 2): h_{x}(0)=0, h_{x}(1)=0
$$

In both cases we impose zero flux of fluid across the boundaries, that is 


$$
\left.\left(\sigma h_{x x x}+\frac{\partial}{\partial x} \Pi(h)+\gamma(x)\right)\right|_{x=0,1}=0 .
$$

The no flux condition implies a fixed volume of fluid, and leads to the volume constraint

$$
\int_{0}^{1} h(x, t) d x=V:=\int_{0}^{1} h(x, 0) d x .
$$

While the primary motivation for our study comes from soap films, the analysis is also relevant to two dimensional liquid films on a solid substrate, i.e. a thin film in a box, which can be modelled with the same basic form of evolution equation. The main purpose of this paper is to analyse the equilibrium solutions and dynamical behaviour of Equation (1.3) on a finite domain, with particular focus on the combined effect of the spatially dependent body force and the DP term.

Pressure relations of the type (1.4) have been considered numerous times. The 6-12 Lennard-Jones potential corresponds to exponents $m=3, n=9[14,19,23]$. Models with $m=3, n=4$ are also common [28, 11,22]. The disjoining/conjoining forces can be thought of separately; a number of studies have included only the conjoining term $[6,8]$, which tends to destabilise the film and lead to rupture [31], in some cases in finite time [36]. The disjoining term serves to stabilise the film and does not permit $h \rightarrow 0$. In the results presented here, we use $m=3, n=4$. Qualitatively, the same basic effects are obtained with the exponents $m=3, n=9$.

In many cases, $h \rightarrow 0$ is not only unphysical but also poses computational issues, and thus understanding the regularizing effect of DP in thin film equations is important from a computational as well as a physical standpoint. Mathematical characterisations of equilibrium solutions to (1.3) with $\gamma(x)=0$ and in the presence of disjoining pres- 
sure are given in $[2,37]$. The global existence and uniqueness of a strictly positive stable equilibrium was proved in [2] for a finite length domain with (BC2) boundary conditions. Stationary solutions to the same system were discussed in [37], in which the set of equilibria was shown to be discrete. These studies however did not include a term $\gamma(x)$ and did not use (BC1) boundary conditions. This is a critical distinction - as we will see, it greatly changes the film behavior and complicates many techniques of analysis. In particular, applications of Sobolev embedding theory in the context of thin film equations often seem to rely on Neumann boundary conditions (see e.g. [1, 2]), and phase plane analysis is rendered impractical by the addition of the non-autonomous function (see Section 3.2 below). Similarly, many prior studies have relied upon the existence of symmetric solutions, which do not exist with the inclusion of $\gamma(x)$.

At the same time, there are numerous studies of (1.3) without DP in the literature (see [7] for a recent review). The majority of these analyses do not contain a non-autonomous function, although a constant $\gamma$ term does often appear. For instance, in the context of draining soap films or flow on a vertical plane, gravity is taken into account with a constant $\gamma$ term $[29,4,3]$. With relation to magnetophoresis, there do not seem to be studies in which the magnetic body force appears in conjunction with the lubrication approximation. Prior to [21], Elias et al. [10] had studied a magnetic soap film and derived a thin film evolution equation, although in their experiments uniform magnetic fields were used and so a spatially dependent body force was not present. There are a few situations where non-autonomous (space-dependent) functions enter the evolution equation. This may be due to Marangoni forcing [9], or to changes in substrate topography [16, 26]; in this latter case, the term $\gamma(x)$ is the third derivative of the shape of the topography feature, 
Effect of disjoining pressure in a thin film equation with non-uniform forcing

so the topography itself can be seen as a non-homogeneous "body force". The steady state dynamics of a thin film on an inclined plane subject to a nonuniform temperature field was studied in $[27,30,17,13]$. And the evolution of a thin film subject to a uniform electric field [35] and flowing over a substrate discontinuity (a step or a trench) was considered in [34].

Thin films on a periodically deformed substrate in the presence of disjoining pressure were analyzed in [33]. In this case, the size of the substrate deformations remained relatively small and the form of the pressure term was different from (1.4), since it admitted a regular expansion as the film thickness went to zero. The analogy between thin films on inclined heterogenous substrates and thin films on the outside of a possibly rotating horizontal cylinder was recently described in [32]. In that work, the disjoining pressure term had the form given by (1.4), with $n=6$ and $m=3$, and the "forcing" corresponded to a cosine function. Finally, horizontal substrates with local chemical heterogeneities, due to localized changes in the parameters appearing in the expression for the disjoining pressure (which was more general than but qualitatively similar to (1.4)) were considered in $[18]$.

Many of the above works were interested in solutions on periodic or infinite domains for the $x$ variable. Central to the present study is therefore the presence of the nonautonomous function $\gamma(x)$, which has order one variations on the interval $[0,1]$ (see Figure 2 ), of the DP $\Pi(h)$, and of a finite domain, associated with given boundary conditions $(\mathrm{BC} 1)$ or $(\mathrm{BC} 2)$, and a zero flux condition at $x=0$ and $x=1$.

This paper is organized as follows: we begin by highlighting the main results of [20], which describes the structure of equilibria of (1.1) - that is (1.3) in the absence of DP 
- for boundary conditions (BC1). In Section 3, we investigate the effect of adding DP, in particular on the structure, regularity, and multiplicity of equilibrium solutions. Due to the function $\gamma(x)$, we are restricted in our analytical treatment of the system. We approach the problem with a combination of analytical arguments and supporting numerical evidence; however we are not able to prove results as in studies that do not include a space-dependent forcing function, especially since the latter cannot be considered as a small perturbation about a uniform forcing, or studies that use Neumann boundary conditions. We show that the structure of solutions with DP is very similar to that without, with two key differences: first, the DP regularises the equilibrium solutions, turning discontinuities in the second derivative in the absence of DP into quick transitions that can be understood in the context of a matched asymptotic expansion with interior layers. Second, our analysis, presented in Section 3.3, suggests that only one stable equilibrium solution of given volume is found in the presence of DP for boundary conditions (BC1). This is in contrast with the case without DP, for which the number of solutions is greater than one and often infinite [20]; moreover, as mentioned above, all of the equilibria that exist without DP appear to be locally attracting. This raises questions about convergence as DP is "turned off", a subject we explore in Section 3.4. Finally, we numerically investigate the effect of the competition between DP and body force in Section 4. For this purpose, we switch to boundary conditions (BC2), which allow coarsening to take place, and we observe how this phenomenon competes with wavelength selection. Conclusions are given in Section 5 . 


\section{Solutions in the absence of disjoining pressure}

We begin by summarizing the results of [20], in which we classified the form of solutions in the absence of the DP term, i.e. in the system

$$
h_{t}+\frac{\partial}{\partial x}\left(\frac{h^{3}}{3}\left(\sigma h_{x x x}+\gamma(x)\right)\right)=0
$$

with (BC1) boundary conditions. This system was analyzed in detail in [20], in which numerical simulations showed that the dynamics evolve, as time goes to infinity, toward a steady state solution which is either a smooth function or part of a continuum of singular solutions. Due to the no-flux condition (1.8), all equilibrium solutions satisfy

$$
\frac{h^{3}}{3}\left(\sigma h_{x x x}+\gamma(x)\right)=0 .
$$

A continuous solution satisfying $\sigma h_{x x x}+\gamma(x)=0$ can easily be found, but it leads to negative values of $h$ in some parameter regimes, which is not physical. However, the above equation also allows for the analytical construction of piecewise solutions, for which $h=0$ over some portion of the region, and $\sigma h_{x x x}+\gamma(x)=0$ over the remainder. These solutions, which are discontinuous in the second derivative of $h$ at a small discrete number of points, were shown in [20] to have an intricate structure. For further discussion, the following features are of importance:

(i) The solution structure can be quite complex, with the number of piecewise regions possible having direct connection with the form of the forcing function $\gamma(x)$.

(ii) Singular solutions exist as part of a continuum family.

(iii) Individual members of the family appear to be locally attracting. More precisely, 
starting with an initial profile that is continuous and close enough to a given member $\mathcal{S}_{e}$ of the family of equilibrium solutions, the dynamics appear to converge toward $\mathcal{S}_{e}$ as $t \rightarrow \infty$. (This may sound surprising given that equilibrium solutions are part of a continuum rather than a discrete set, which in turn would suggest that each particular solution should be neutrally stable, but this argument does not hold here because the equilibrium solutions in question are discontinuous in their second derivative.)

(iv) Discontinuities in $h_{x x}$ exist at some or all of the piecewise junctions.

The combination of (ii) and (iii) means that initial conditions play an important role in the film evolution and eventual equilibrium state. Underlying these results is a simple counting argument. Consider, for example, the solution depicted in Figure 3. The functions $h_{1}$ and $h_{2}$ each satisfy $\sigma h_{x x x}+\gamma(x)=0$, and are connected by a region where $h=0$. In the construction of the piecewise solution, there are 3 constants of integration for each function $h_{1}$ and $h_{2}$. The junction points $x_{1}$ and $x_{2}$ are unknown - this gives 8 total unknowns. Imposing (BC1), the volume constraint, and continuity of $h$ and $h_{x}$ is 7 conditions. Thus continuity of $h_{x x}$ cannot in general be imposed at both $x_{i}$. But there is still a free variable, so that the $x_{i}$ live on an implicit curve; this leads to a continuum of solutions, all with discontinuous second derivative, such that the endpoints of the continuum have $h_{x x}$ discontinuous at only one of the $x_{i}$. Similar considerations apply for solutions with "bubbles" of non-zero $h$ in the $\left[x_{1}, x_{2}\right]$ interval, as discussed in [20].

\section{Effect of disjoining pressure}

The addition of the DP term has several important consequences for the form and structure of equilibrium solutions, as well as for the dynamical evolution to the equilibrium 


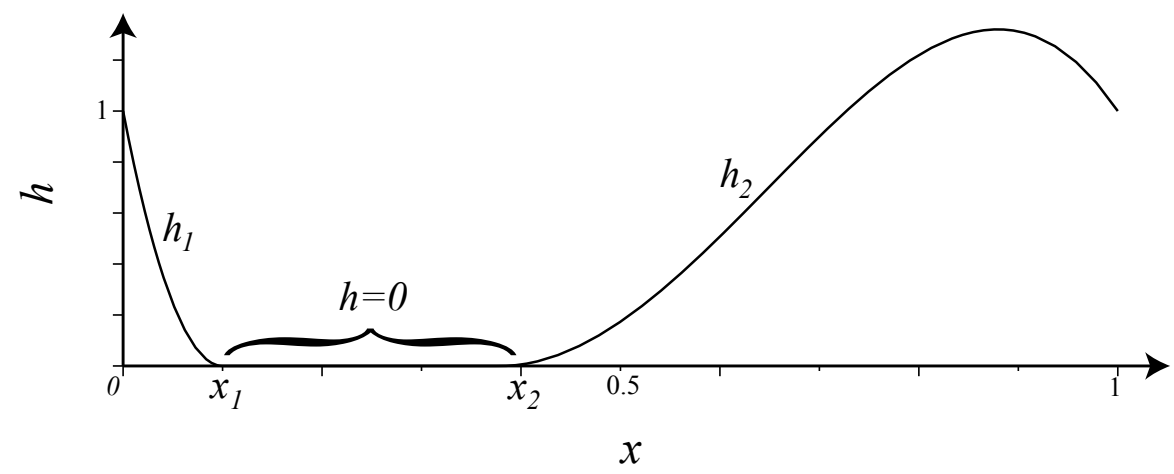

FIGURE 3. Depiction of a piecewise equilibrium solution in the case of no DP and (BC1) boundary conditions. Two regions where $h$ is non-zero connect to a region where $h=0$ at the points $x_{1}$ and $x_{2}$.

state. In Figure 4 we plot the equilibrium profiles of $h, h_{x}$, and $h_{x x}$ with and without DP, obtained by numerically integrating Equation (1.3) to large time $\left(t=10^{7}\right.$ ) (our numerical approach is outlined in [20]). In this example, $\sigma=0.004$, the volume $V=0.5$, and $\gamma(x)$ takes the form (1.2) with $\lambda=1$. Boundary conditions (BC1) were used, and in the DP term, $A=1$, and $h^{*}=0.05$. In both cases, a parabolic initial profile was chosen. The dashed lines are the final profiles for simulations with no DP $(A=0)$; the solid lines are the final profiles with the DP. For the initial conditions used, the system without DP tends to a piecewise profile with a single discontinuity in the second derivative. Other members of the family of singular solutions described in [20] typically have two discontinuities in $h_{x x}$. The profile with $A \neq 0$ has a very similar form, with a few key differences: as stated, the DP keeps $h$ strictly positive (top panel of Figure 4). Observe also that the DP has regularized the solution, which now has a continuous second derivative, as shown in the bottom panel of Figure 4. It is moreover interesting to note that the single 
discontinuity in $h_{x x}$ in the case $A=0$ is replaced by two locations with rapid change in $h_{x x}$, which however remains continuous. Our goal in this section is to understand the structure of equilibrium solutions to (1.3) with (BC1) boundary conditions in the presence of DP and of a non-perturbative body force $\gamma(x)$, as well as how these solutions evolve as the DP goes to zero.

\subsection{Energy considerations}

Before we construct equilibrium solutions, we first briefly discuss the variational properties of (1.3). Assume that $h(x, t)$ is smooth and positive, and let

$$
E=\int_{0}^{1}\left(\frac{\sigma}{2} h_{x}^{2}-P(h)-h \Gamma(x)\right) d x
$$

where $d P / d h=\Pi(h)$ and $d \Gamma / d x=\gamma(x)$. With boundary conditions $(\mathrm{BC} 1), h(0)=$ $h(1)=1$, the Fréchet derivative of $E$ is given by

$$
\frac{\delta E}{\delta h}=-\left(\sigma h_{x x}+\Pi(h)+\Gamma(x)\right) \equiv Q(h, x),
$$

and the thin film equation (1.3) reads

$$
h_{t}=\frac{\partial}{\partial x}\left(\frac{h^{3}}{3} \frac{\partial}{\partial x} Q(h, x)\right)=\frac{\partial}{\partial x}\left(\frac{h^{3}}{3} \frac{\partial}{\partial x}\left(\frac{\delta E}{\delta h}\right)\right) .
$$

Using this expression and integrating by parts thus gives

$$
\int_{0}^{1} Q(h, x) h_{t} d x=\left[\frac{h^{3}}{3}\left(\frac{\partial}{\partial x} Q(h, x)\right) Q(h, x)\right]_{0}^{1}-\int_{0}^{1} \frac{h^{3}}{3}\left(\frac{\partial}{\partial x} Q(h, x)\right)^{2} d x,
$$

which simplifies to

$$
\int_{0}^{1} Q(h, x) h_{t} d x=-\int_{0}^{1} \frac{h^{3}}{3}\left(\frac{\partial}{\partial x} Q(h, x)\right)^{2} d x \leq 0,
$$

since we assume that $\frac{\partial}{\partial x} Q(h, x)$ vanishes at the endpoints and that $h$ remains positive. 

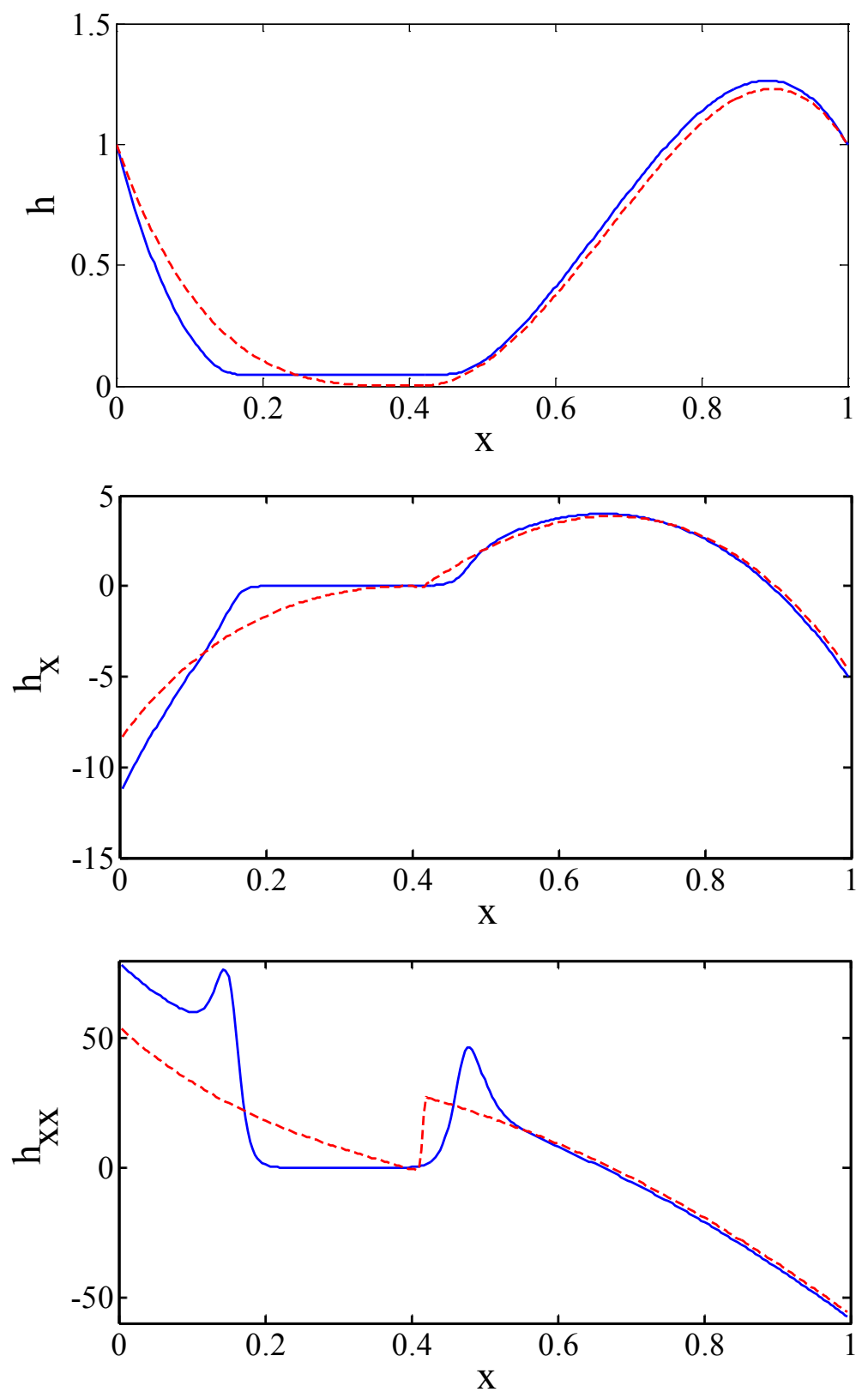

FiguRE 4. Equilibrium profiles of $h, h_{x}$, and $h_{x x}$ for the system without DP (dashed curves) and with DP (solid curves), with (BC1) boundary conditions. Here $\lambda=1, \sigma=0.004, V=0.5$, and for the solid curves $A=1, h^{*}=0.05$. 
Following [24], one can also calculate

$$
\begin{aligned}
\int_{0}^{1} Q(h, x) h_{t} d x & =\left[-\sigma h_{x} h_{t}\right]_{0}^{1}-\frac{d}{d t} \int_{0}^{1}\left(\frac{-\sigma}{2} h_{x}^{2}+P(h)+h \Gamma(x)\right) d x \\
& =-\frac{d}{d t} \int_{0}^{1}\left(\frac{-\sigma}{2} h_{x}^{2}+P(h)+h \Gamma(x)\right)
\end{aligned}
$$

where we have used $h_{t}(0)=h_{t}(1)=0$. Therefore,

$$
\frac{d E}{d t}=-\frac{d}{d t} \int_{0}^{1}\left(\frac{-\sigma}{2} h_{x}^{2}+P(h)+h \Gamma(x)\right)=\int_{0}^{1} Q(h, x) h_{t} d x \leq 0
$$

i.e. the functional $E$ decreases as a function of time under the thin film dynamics. If we assume that the solutions $h(x, t)$ remain regular for smooth initial data, since the three terms in the expression of $E$ are bounded from below, one may expect $E$ to reach a minimum value subject to the constant volume constraint given by (1.9). To show that the energy $E$ is bounded from below, we note that the function

$$
P(h)=A h^{*}\left(\frac{1}{m-1}\left(\frac{h^{*}}{h}\right)^{m-1}-\frac{1}{n-1}\left(\frac{h^{*}}{h}\right)^{n-1}\right)
$$

reaches its maximum at $h=h^{*}$, so that

$$
\begin{aligned}
E & =\int_{0}^{1}\left(\frac{\sigma}{2} h_{x}^{2}-P(h)-h \Gamma(x)\right) d x \\
& \geq \int_{0}^{1}(-P(h)-h \Gamma(x)) d x \geq \int_{0}^{1}\left(-P\left(h^{*}\right)-h \Gamma(x)\right) d x \\
& \geq-P\left(h^{*}\right)+V m
\end{aligned}
$$

where $m$ is the minimum of $-\Gamma(x)$ on $[0,1]$. Clearly the values of $m$ and $P\left(h^{*}\right)$ depend on arbitrary constants of integration, but once these are fixed, they define a lower bound for $E$. To fix the ideas, a plot of

$$
\Gamma(x)=x+\frac{\lambda}{2\left(1+x^{2}\right)^{3}}
$$

is shown in Figure 2 for $\lambda=1$. 
Minimization of $E-C \int_{0}^{1}(h(x)-V) d x$ leads to $\frac{\delta E}{\delta h}=C$, where $C$ is the Lagrange multiplier associated with the volume constraint. This equation reads $Q(h, x)=C$ and is equivalent to $\frac{d}{d x} Q(h, x)=0$, i.e. to

$$
\sigma h_{x x x}+\Pi_{x}+\gamma(x)=0
$$

It is beyond the scope of this paper to prove existence and regularity of solutions to (1.3). In particular, it is not clear that the treatment of [2] may be extended to the present situation because of the boundary conditions (BC1). If one assumes that $h(x, t)$ remains in $H^{1}([0,1])$, then decay of the functional $E$ leads to control of the $H^{1}$ norm of $h$. Moreover, if $h(x, t)$ is also Hölder continuous with exponent $1 / 2$ on $[0,1]$, a proof similar to that of Theorem 1 of [2] indicates that $h$ remains positive and bounded. This implies that the disjoining pressure is regularizing the solution. Theorem 2 of [2] may also be extended to the present situation to show the existence of a global minimizer in $H^{1}([0,1])$ subject to the volume constraint, and that the minimizer is positive almost everywhere in $[0,1]$. But boundary conditions $(\mathrm{BC} 1)$ prevent the extension by zero of $h(x, t)$ to a function in $H^{1}(\mathbb{R})$, thereby complicating further analysis. In what follows, we assume that equilibrium solutions of (1.3) exist, note that they satisfy

$$
\frac{h^{3}}{3}\left(\sigma h_{x x x}+\Pi_{x}+\gamma(x)\right)=\mathcal{C},
$$

and that the integration constant $\mathcal{C}$ must be zero because of the no-flux boundary condition. Since $\Pi(h)$ becomes infinite as $h \rightarrow 0$, piecewise solutions with $h=0$ over a portion of the domain are not possible, and equilibrium solution must thus satisfy (3.2).

We have checked that the energy $E$ decreases as a function of time in our numerical 
simulations of (1.3) and, as expected, that it remains above the lower bound $-P\left(h^{*}\right)+$ $V m$.

\subsection{Equilibrium solutions: matched asymptotic expansion}

In this section, we construct equilibrium solutions to (1.3) with (BC1) boundary conditions, that is we look for solutions to (3.2) with $h(0)=h(1)=1$. As mentioned above, one integration can be performed leading to a second order system of the form $Q(h, x)=C$. Multiplying by $h_{x}$ and integrating again, as is done in [19,2], then reduces the problem to a first order integro-differential system, but a phase plane type analysis is still three dimensional due to the space dependence of $\Gamma(x)$. Written as a third-order dynamical system, $Q(h, x)=C$ reads

$$
\frac{d h}{d t}=u, \quad \frac{d u}{d t}=\frac{-1}{\sigma}(\Pi(h)+\Gamma(x)+C), \quad \frac{d x}{d t}=1 .
$$

The asymptotic solution (solid curve) of the simulation of Figure 4 is shown in the three-dimensional phase space of (3.3) in Figure 5. The arrows represent the vector field along the trajectory. Also plotted are the projections of the trajectory on the $x=0$, $h_{x}=-12$, and $h=1.5$ planes. The dotted curves show an almost identical trajectory obtained as a numerical solution of (3.3) with $C=-0.82982$. It can be seen that both trajectories go through a narrow "vertical tubular region" where $h$ is near $h^{*}$ and $h_{x}$ is near 0 . If a trajectory of (3.3) fails to enter this region, it typically reaches $h=0$ in finite time with $h_{x}$ diverging to $-\infty$; if it leaves the narrow tubular at a slightly different point, then $h$ typically diverges to $+\infty$. As a consequence, numerical integration of (3.3) near equilibrium solutions of (1.3) that are of interest for this study is very sensitive to the choice of initial conditions in the phase space of (3.3). This is not a problem 


$$
\mathrm{C}=-0.82982
$$

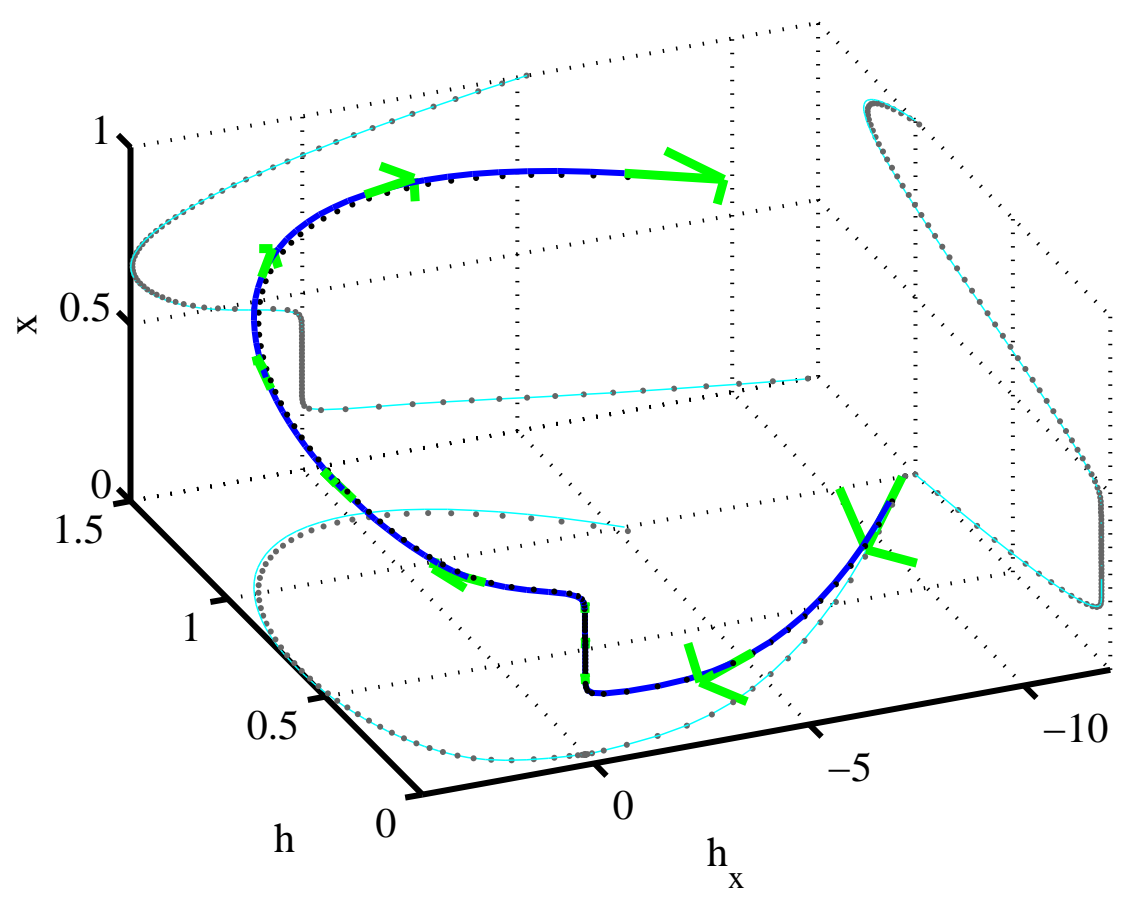

Figure 5. Phase space for the dynamical system given by (3.3). The solid curve is the solution of (1.3) shown in Figure 4. The dotted curve is a solution of (3.3) with $C=-0.82982$. The arrows show the dynamical system vector field along these curves. Also shown are the projections of these trajectories on planes parallel to the coordinate planes. Parameters are $\lambda=1, \sigma=0.004$, $V=0.5, A=1, h^{*}=0.05$.

for solutions of larger volume, for which $h$ does not "spend as much time" near $h^{*}$. A numerical exploration of the dynamics of (3.3) shows that one can find a curve in the $\left(C, h_{x}(0)\right)$ plane, such that the solution of (3.3) with the chosen value of $C$ and initial conditions $h(0)=1, u(0)=h_{x}(0), x(0)=0$ is such that $h(1)$ is near 1 . Each point on this curve therefore corresponds to an equilibrium solution of (1.3), but the volume of the solution changes as one moves along the curve. Based on this numerical exploration, and keeping in mind the intrinsic limitations mentioned above, it therefore appears that for 


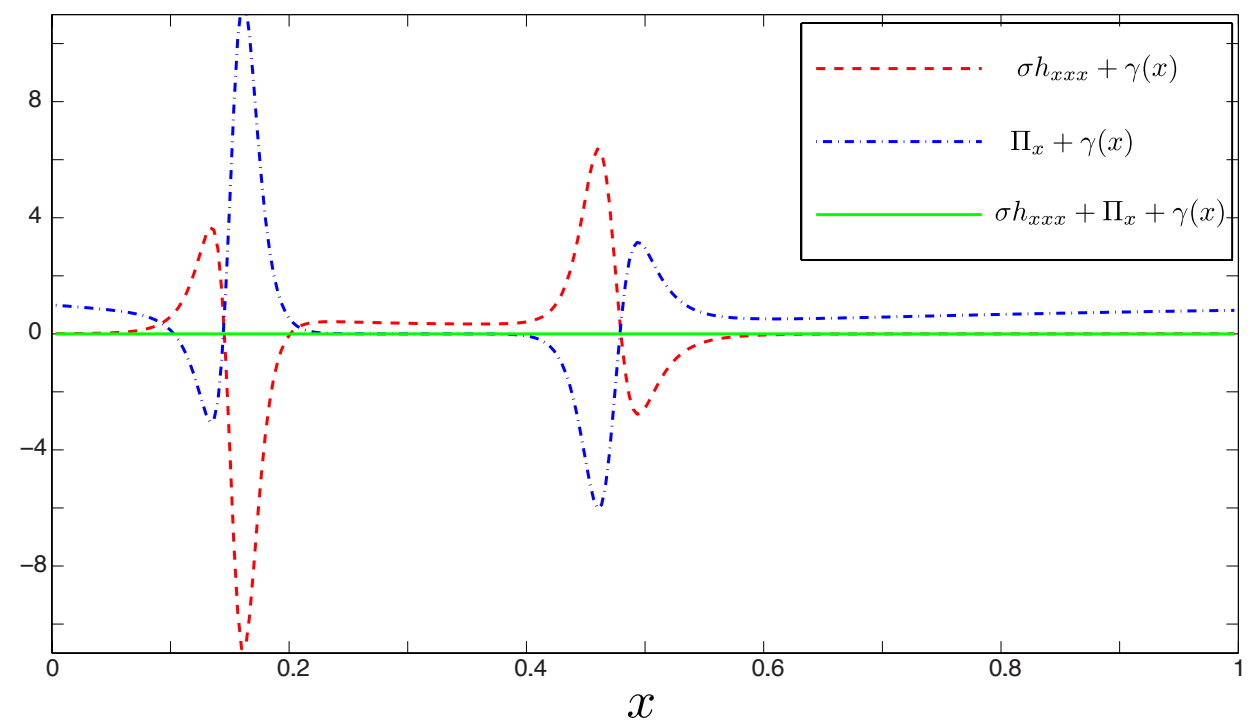

FIGURE 6. Decomposition of the equilibrium solution with DP into components. This provides a natural division of the spatial domain into 3 regions.

the parameter ranges of interest in this paper there exists only one equilibrium solution to (1.3) with (BC1) boundary conditions. As indicated above, an analytical investigation of the existence and uniqueness of this solution is beyond the scope of this paper.

We now take a different approach to obtain these solutions, which consists in looking for solutions to (3.2) using matched asymptotic expansions. We can make progress analytically by recognizing the relative magnitudes of the contributions of the various terms in this equation: note that the first term is a capillary term, the second term is the DP, and the third term is the forcing term. When $h$ is close to zero, the solution tends to be very nearly flat, and so the capillary term has little contribution, whereas the DP term is significant; here the solution is determined by a balance between the DP and forcing terms. On the other hand, when $h$ is far from zero, the DP has very small contribution, and the solution is determined by the balance between the capillary and forcing terms. 


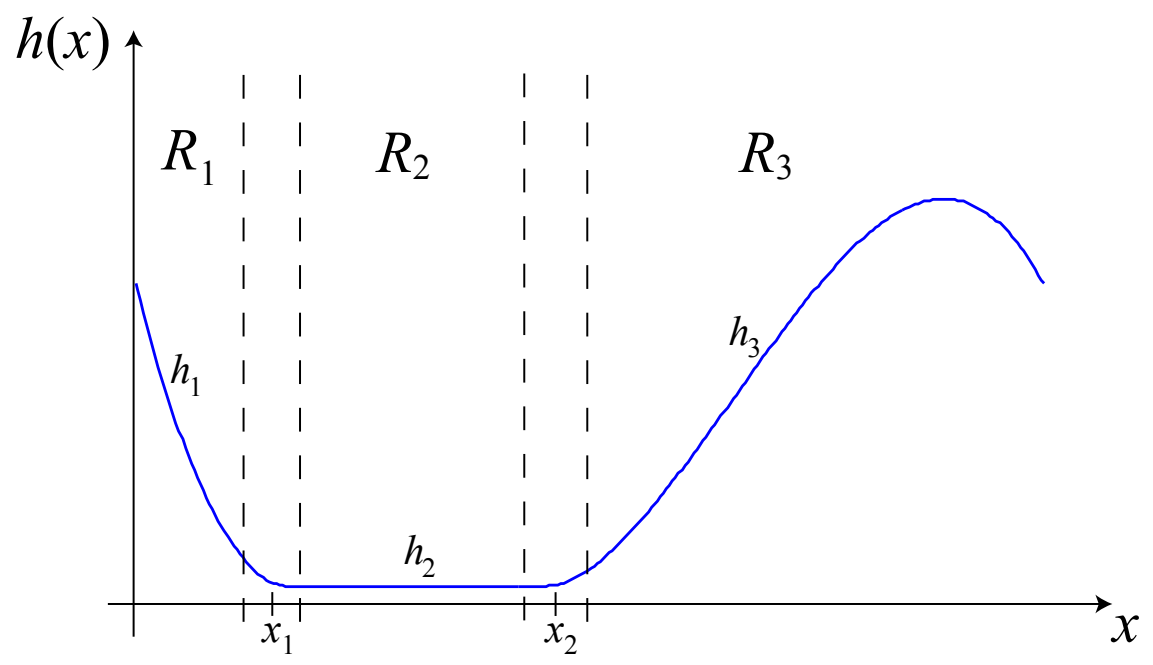

FiguRE 7. Setup of three regions with two interior layers for a matched asymptotic expansion for equilibrium solutions with DP and (BC1) boundary conditions.

To demonstrate, in Figure 6 we take the numerical equilibrium solution with $A=1$ plotted in Figure 4, and plot the various components of Equation (3.2). The red (dashed) curve is the sum of the capillary and forcing terms, the blue (dash-dotted) curve is the sum of the DP and forcing terms, and the green (solid) curve is the sum of all 3 terms. Observe that the green curve is zero over the entire domain, confirming that the thin film is in equilibrium. The red and blue curves divide the domain into 3 distinct regions. On the outsides, the red curve is nearly zero, while in the middle the blue curve vanishes. Between the regions, neither red nor blue is zero, signifying a transition in the balance. 


\subsubsection{Outer regions}

Though we have only illustrated the decomposition for a particular set of parameters and particular forcing function $\gamma(x)$, the structure is found to occur generally. This suggests that, assuming they exist, equilibrium solutions with DP could generally be constructed through a matched asymptotic expansion, with interior layers. To form the outer solution, we define $\epsilon:=h^{*}$ to be a small parameter, and divide the domain into 3 regions, depicted in Figure 7. Note that the locations of the boundaries between the regions is not known a priori. In regions $R_{1}$ and $R_{3}, h$ is $\mathrm{O}(1)$ and so the $\mathrm{DP}$ is negligible. The same basic idea, that the DP is negligible on the mesoscale, is utilized in an asymptotic expansion for a moving droplet in [25]. The first order solution in regions $R_{1}, R_{3}$ satisfies

$$
\sigma h^{\prime \prime \prime}(x)+\gamma(x)=0,
$$

with boundary condition $h_{1}(0)=1$ in $R_{1}$ and $h_{3}(1)=1$ in $R_{3}$. This indicates that the product $\sigma h^{\prime \prime \prime}(x)$ is of order one and balances the forcing $\gamma$; as a consequence, smaller values of $\sigma$ will be associated with larger values of $h^{\prime \prime \prime}(x)$. In $R_{2}$, we assume $h_{2}$ is $\mathrm{O}(\epsilon)$. By defining $g=h / \epsilon$, the system becomes

$$
\epsilon \sigma g_{x x x}+A \frac{g_{x}}{g}\left(\frac{m}{g^{m}}-\frac{n}{g^{n}}\right)+\gamma(x)=0
$$

Hence in $R_{2}$ the $g_{x x x}$ term does not appear to first order. Dropping the first term and integrating once, we obtain

$$
A\left(\frac{1}{g^{n}}-\frac{1}{g^{m}}\right)+\Gamma(x)=\tilde{C}
$$


where $\tilde{C}$ is a constant. We cannot generally solve for $g$ exactly, and so the solution $h_{2}$ in this region is only determined implicitly.

\subsubsection{Interior layers}

As is apparent in Figure 4, the interior layers are characterized by a rapid change in the second derivative of $h$. For the layer located at $x_{i}$ (note the $x_{i}$ are not intially known), define the stretched variable

$$
\bar{x}=\frac{x-x_{i}}{\epsilon^{p}}, \quad p>0 .
$$

Let $H(\bar{x})$ designate the solution in this region, and take $H \sim \epsilon^{r} H_{0}+\ldots$ Writing $\sigma=\epsilon^{q}$ ( $q$ is assumed known and positive), substitution into the full system (1.3) yields

$$
\begin{aligned}
& \epsilon^{q-3 p+r} H_{0}^{\prime \prime \prime}(\bar{x}) \\
& +\epsilon^{-p+m-m r} A \frac{H_{0}^{\prime}}{H_{0}}\left(\frac{m}{H_{0}^{m}}-\epsilon^{(n-m)(1-r)} \frac{n}{H_{0}^{n}}\right) \\
& +\gamma\left(x_{i}+\epsilon^{p} \bar{x}\right)=0 .
\end{aligned}
$$

We now expand $\gamma\left(x_{i}+\epsilon^{p} \bar{x}\right) \sim \gamma\left(x_{i}\right)+\mathrm{O}\left(\epsilon^{p}\right)$ and assume $\gamma\left(x_{i}\right)=O(1)$. We also assume (and have numerically checked by plotting the value of $h$ at the core of each layer as a function of $\left.h^{*}=\epsilon\right)$ that $r=1$. Multiplying the above equation by $\epsilon^{3 p-q-1}$ gives

$$
\begin{aligned}
& H_{0}^{\prime \prime \prime}(\bar{x}) \\
& +\epsilon^{2 p-q-1} A \frac{H_{0}^{\prime}}{H_{0}}\left(\frac{m}{H_{0}^{m}}-\frac{n}{H_{0}^{n}}\right) \\
& +\epsilon^{3 p-q-1} \gamma\left(x_{i}\right)+O\left(\epsilon^{4 p-q-1}\right)=0 .
\end{aligned}
$$

Since $p>0$, the last two terms are of higher order and to balance the equation at lowest order, we need to set $2 p-q=1$. Thus, the dominant terms in the interior layers are the capillary and DP terms. The lowest order solution should thus result from balancing 
these two terms, with the effect of the forcing coming at higher order. This is in line with the observation that in the transition from $R_{1}$ to $R_{2}, h_{x x}$ and $\Pi(h)$ both vary quickly, implying large contributions from $h_{x x x}$ and $\Pi_{x}$ in the interior layers. The solution $H_{0}$ then satisfies

$$
H_{0}^{\prime \prime \prime}(\bar{x})+A \frac{H_{0}^{\prime}}{H_{0}}\left(\frac{m}{H_{0}^{m}}-\frac{n}{H_{0}^{n}}\right)=0,
$$

which can in principle be solved for $H_{0}$.

\subsubsection{Matching}

Having solved for the three outer solutions and the two interior layer solutions, the integration constants as well as the locations of the layers are determined through matching. Ideally, this would be done analytically, rendering explicit formulas. However, due to the nonlinearity of the $\Pi_{x}$ term, which appears at lowest order both in region $R_{2}$ and in the interior layers, together with the implicit representation of $h_{2}$, the matching is impractical analytically. Nevertheless, we can do the matching numerically and check the validity of the matched asymptotic expansion. Suppose all parameters: $\lambda, \sigma, A, V$, and $h^{*}=\epsilon \ll 1$, are fixed. We also take as known the width of each layer, which can be approximated by estimating the width of the transition layer in an equilibrium decomposition such as Figure 6.

For given parameters, our approach is as follows: Let $\tilde{C}$, the constant of integration in $R_{2}$, and $x_{1}$ and $x_{2}$, the locations of the layers, be shooting parameters. Appropriate initial guesses for these may be obtained from a full numerical solution. For given $x_{1}$ and $x_{2}$, matching the solution in the outer regions in principle leads to 4 conditions, two 

at each of the $x_{i}$ 's. For the numerical approximation, we replace this by matching at 4 locations given by $x=x_{m}=x_{i} \pm \Delta x_{i} / 2, i=1,2$, where $\Delta x_{i}$ is the width of the $i$ th layer. In terms of the interior layers variables, the matching, which in principle should occur at $\pm \infty$, is replaced by a numerical condition at $\bar{x}=\bar{x}_{m}= \pm \Delta x_{i} /\left(2 \epsilon^{p}\right)$.

We start in the middle region $R_{2}$ and work outwards. For fixed $\tilde{C}$, Equation (3.6) can be solved for the value of $g$ at $x=x_{m}$ corresponding to the inner boundaries; $g^{\prime}$ is then found from

$$
g^{\prime}(x)=-\frac{\gamma(x)}{\Pi^{\prime}(g)}
$$

where $\Pi(g)=A\left(g^{-n}-g^{-m}\right)$, and then

$$
g^{\prime \prime}(x)=-\frac{\gamma^{\prime}(x)+\Pi^{\prime \prime}(g) g^{\prime 2}}{\Pi^{\prime}(g)} .
$$

Appropriately accounting for the change of variables, the values of $g, g^{\prime}$, and $g^{\prime \prime}$ at the $x_{m}$ are translated to values of $H_{0}, H_{0}^{\prime}$, and $H_{0}^{\prime \prime}$ at the $\bar{x}_{m}$. We use these conditions to solve Equation (3.10) as an initial value problem, integrating from the inner to the outer edge of the interior layers. At the outer edge of the layers, i.e. the boundaries between the interior layers and the regions $R_{1}$ and $R_{3}$, the values of $H_{0}, H_{0}^{\prime}$, and $H_{0}^{\prime \prime}$ are translated into boundary conditions for $h_{i}, h_{i}^{\prime}$, and $h_{i}^{\prime \prime}, i=1,3$. Integrating Equation (3.4) and imposing these conditions, the functions $h_{1}$ and $h_{3}$ can be found exactly, and the solution profile is fully determined. We then form the vector

$$
\mathbf{F}=\left[\begin{array}{c}
h_{1}(0)-1 \\
h_{3}(1)-1 \\
\int_{0}^{1} h(x) d x-V
\end{array}\right]
$$


and iterate on $\mathbf{z}=\left(x_{1}, x_{2}, \tilde{C}\right)^{T}$ until $\|\mathbf{F}(\mathbf{z})\|$ is less than a small fixed tolerance. Computing the Jacobian is particularly difficult and expensive for this system, and so we used Broyden's method [5] to perform the iteration, which is a quasi-Newton method in which the Jacobian must be computed only for the first iteration. In Figure 8, we plot a sample comparison between the full numerical solution (found by numerical integration of the full system up to large time) and the matched asymptotic expansion obtained by the numerical approach described above, using the same parameter values as in Figure 4 (in particular, note that $\epsilon=h^{*}=0.05$ ). The curves are nearly indistinguishable in the function and first derivative, with a small difference appearing in the interior layers in the second derivative. Included at the bottom of Figure 8 is the same comparison in the second derivative, but with $h^{*}=0.005$. Here the match is much closer, illustrating as expected that as $h^{*}$ goes to zero, the matching improves. The slight discrepancy in the bottom plot is partially attributable to the uncertainty in the exact layer width, thus in this numerical approach where exactly to impose the matching is not explicitly defined. Nevertheless, the close similarity verifies the validity of the matched asymptotic expansion.

\subsubsection{Layer width}

Key to the numerical matching approach outlined above is knowledge of the width of the interior layers, which we denoted $\Delta x$. The relationship $2 p-q=1$ suggests that the quantity $(\Delta x)^{2} / \sigma$ should vary linearly with $\epsilon=h^{*}$. We can check this numerically. Rather than letting Equation (1.3) evolve until a steady state solution is reached for different values of $h^{*}$, we find equilibrium solutions to Equation (3.2) by means of a 

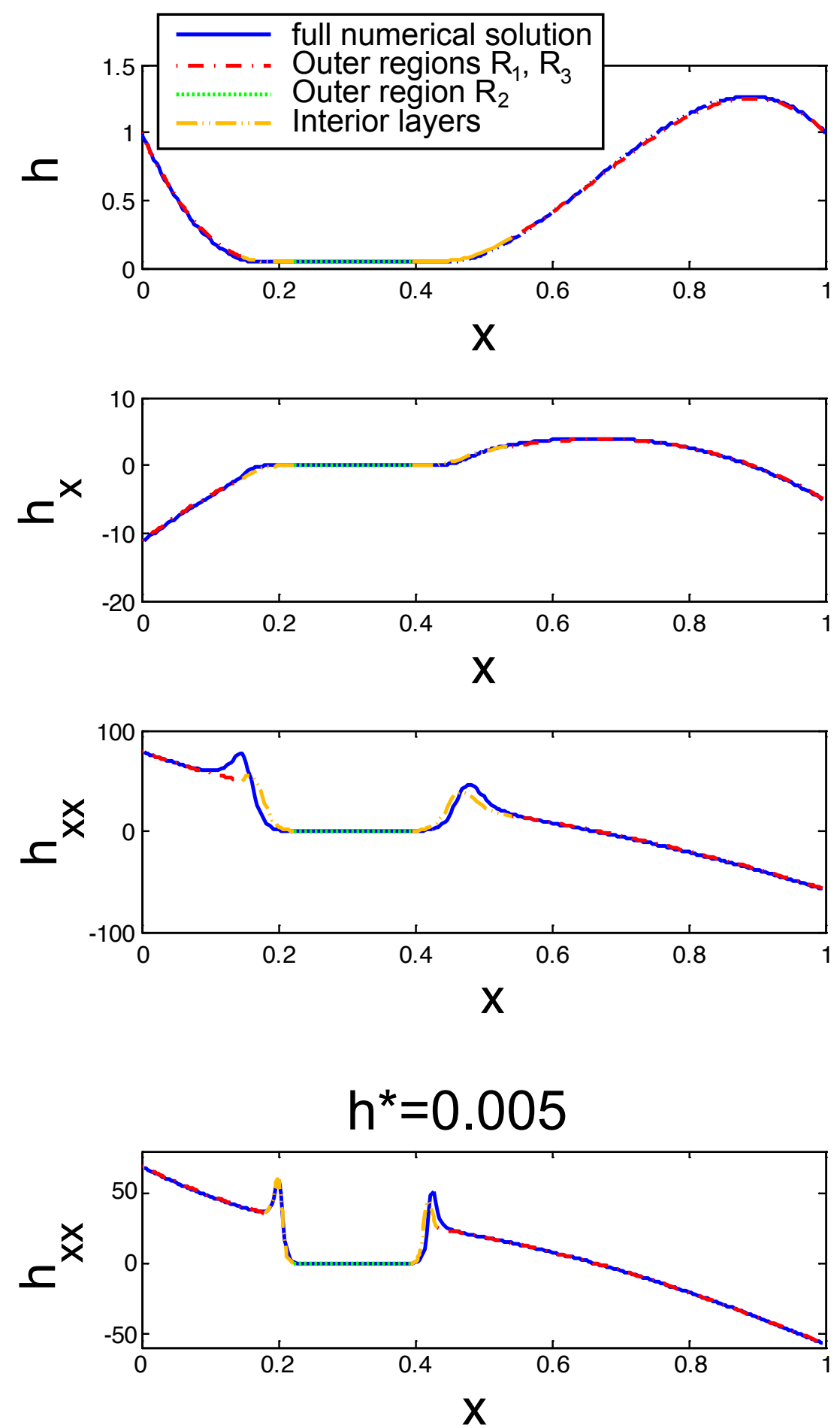

Figure 8. Comparison of equilibrium solution with DP and (BC1) boundary conditions as computed through the matched asymptotic expansion, and the full numerical solution. For the matched asymptotic expansion solution, different colors and linestyles are used for the solution in each different region to highlight the transition, which is clearly seen in the second derivative $h_{x x}$. Parameters are $\lambda=1, \sigma=0.004, A=1, V=0.5$. For the top 3 plots, $h^{*}=0.05$; the bottom plot shows just the second derivative for $h^{*}=0.005$. 


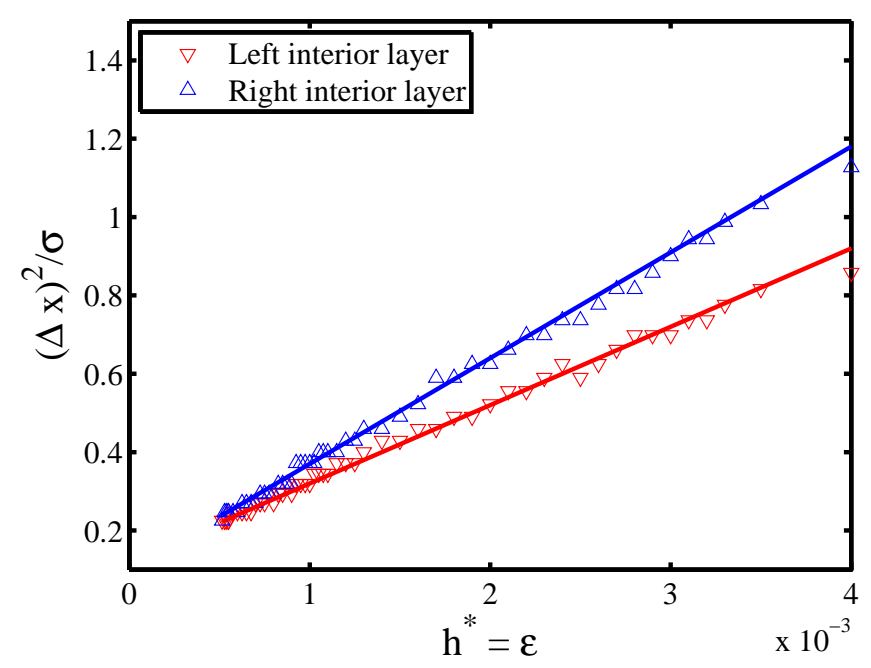

Figure 9. Quantity $(\Delta x)^{2} / \sigma$ for the left (down triangles) and right (up triangles) interior layers, as a function of $h^{*}=\epsilon$. The width $\Delta x$ of each layer is estimated from the equilibrium profile obtained by Newton-Raphson iterations for each value of $h^{*}$. The solid lines are drawn to guide the eye and have equation $y=0.12+200 \epsilon$ (left layer) and $y=0.10+270 \epsilon$ (right layer). Newton-Raphson method, which takes into account both boundary conditions, as well as the volume constraint. As an initial profile for the iterations at $h^{*}=0.05$, we take the steady-state solution obtained by numerical integration of Equation (1.3) until a large time, $t=300,000$ specifically. All other parameters are the same as in the previous figures. Then, we increase or decrease $h^{*}$ by small steps, using the solution at one value of $h^{*}$ as the initial condition for the Newton-Raphson iterations at the next value. We define the start of the left layer as the point where $\sigma h_{x x x}+\gamma(x)$ (the red dashed curve in Figure 6) becomes greater in absolute value than a fixed threshold (set to 0.05), and the end of the layer as the point where $\Pi_{x}+\gamma(x)$ (the blue dash-dotted curve) drops, in absolute value, below the same threshold value, with a similar process defining the right layer. The width $\Delta x$ of each layer is the difference between the starting and ending 
Effect of disjoining pressure in a thin film equation with non-uniform forcing

points. In Figure 9, the quantities $(\Delta x)^{2} / \sigma$ are plotted as functions of $h^{*}$ for the left (down triangles) and right (up triangles) interior layers. The solid lines are drawn to guide the eye and have equations $y=0.12+200 \epsilon$ (left) and $y=0.10+270 \epsilon$ (right). The behavior of both quantities is reasonably linear for small values of $h^{*}$, and departs from linearity (not shown) at higher values of $h^{*}$.

\subsection{Uniqueness}

The matched asymptotic expansion approach demonstrates that solutions to Equation (3.2) with boundary conditions (BC1) have a structure defined by regions in space where two of the three terms in this equation are dominant. In particular, the second derivative of $h$ and the DP term $\Pi(h)$ vary strongly in two interiors layer, whose width $\Delta x$ is such that $(\Delta x)^{2} / \sigma$ scales like $h^{*}$. Moreover, equilibrium solutions with DP have a similar profile as those without DP, with a key distinction being the regularity of solutions with DP. The other key distinction involves the multiplicity of solutions, and can be explained by a variable/condition count. As mentioned, without DP the system is one constant short of imposing continuity of $h_{x x}$ at both $x_{i}$, thus either continuity is imposed at only one of the $x_{i}$ or else the free constant leads to a family of solutions with $h_{x x}$ discontinuous at both $x_{i}$. In the system with DP, there is an extra free variable - which one may think of as the integration constant in the middle region $R_{2}$. Hence, continuity of $h_{x x}$ can in principle be imposed at both $x_{i}$, so the discontinuity without DP is transformed into a quick, but continuous, transition.

Further, the variable count in the matched asymptotic expansion process is such that the number of unknowns matches the number of conditions, and so equilibrium solutions 


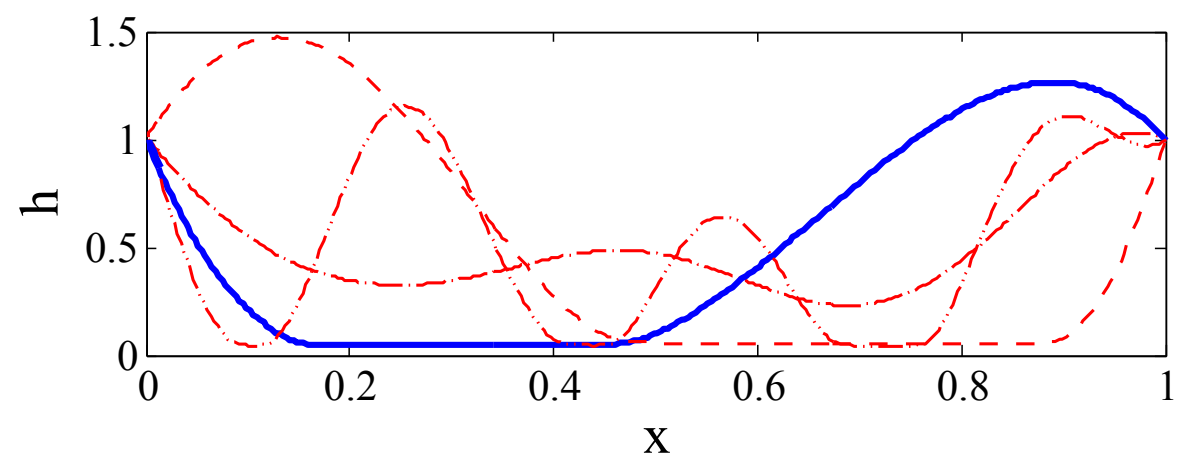

Figure 10. Initial (dashed) and final (solid) film profiles for (BC1) boundary conditions and parameters $A=1, h^{*}=0.05, \sigma=0.004, V=0.5$, and $\gamma$ given by Equation (1.2) with $\lambda=1$. should form at most a discrete set. Indeed, at lowest order, there are 3 constants of integration in each of the regions $\mathrm{R} 1, \mathrm{R} 3$, and the two interior layers, and one constant of integration in R2. Combined with the unknown locations of the layers, $x_{1}$ and $x_{2}$, this is a total of 15 unknowns. Matching the interior layers to each of the outer regions gives 12 conditions, plus the two boundary conditions at $x=0$ and $x=1$ and the volume constraint gives 15 conditions. Thus, matching should in principle determine all solutions at lowest order and the locations of the layers. Based on the numerical matching, this seems to be the case.

While the counting argument indicates at most a discrete set of solutions, the numerical exploration of the dynamical system (3.3) and our numerical simulations of Equation (1.3) have suggested an even stronger conjecture: that there is at most one steady-state solution in the system with DP and (BC1), and that this solution, which minimizes the energy functional $E$ subject to the volume constraint, is in fact stable. Indeed, all of the simulations we have run show that a single stable profile is selected by the dynamics, for 
Effect of disjoining pressure in a thin film equation with non-uniform forcing

given values of the parameters. An example of such a numerical experiment is provided in Figure 10, where the initial and final profiles are plotted for the parameters as in Figure 4. Even for vastly different initial profiles (but all with volume $V=0.5$ ), the equilibrium solution of Figure 4 (which was obtained starting from a parabolic profile) is approached. Moreoever, in our numerical implementation of the Newton-Raphson method, we have found that only a single equilibrium solution is selected for each value of $h^{*}$, although it should be noted that the method requires an initial profile fairly close to the equilibrium solution to converge. Combined with the numerical exploration of the dynamical system (3.3), these numerical experiments lend strong support to the hypothesis that in the presence of DP there is exactly one stable steady-state solution of given volume to (3.2) with boundary conditions (BC1).

\subsection{Asymptotic behavior}

That a family of solutions exists without DP and at most a discrete set (and likely a single solution) of given volume exists with DP raises the following questions: What happens as the disjoining pressure is "turned off"? Does the sequence of solutions with DP converge? If so, does the asymptotic solution solve the equation in the absence of DP, i.e. is it a member of the continuum of solutions discussed in section 2? And if so, which one? In this section we numerically explore the behavior of equilibrium solutions in the limits $h^{*} \rightarrow 0$ and $A \rightarrow 0$. 
3.4.1 As $h^{*} \rightarrow 0$

To explore whether solutions with DP are converging to a particular solution without DP, it is necessary to determine the locations $x_{1}$ and $x_{2}$ of the interior layers, which we define as the points halfway between the left and right end-points of each layer. Figure 11 plots the values of $x_{1}$ and $x_{2}$ for the solutions obtained by Newton-Raphson iterations, as $h^{*} \rightarrow 0$. The error bars indicate the width $\Delta x$ of each layer. The two points marked by a star at $h^{*}=0$ have vertical coordinates $x_{1}=0.229$ and $x_{2}=0.402$, and correspond to the points of discontinuity in $h_{x x}$ of a solution, referred to below as $h_{0}$, that exists in the absence of DP. The existence of branches of singular solutions when DP $=0$ is briefly justified at the end of Section 2 ; the values of $x_{1}$ and $x_{2}$ along each branch may be estimated numerically, as explained in [20]. The fact that $x_{1}$ and $x_{2}$ appear to converge to values that are on the branch of solutions in the absence of DP suggests that the process of sending $h^{*}$ to zero may select a particular member of the continuum of steady-state solutions of Equation (1.3) in the absence of DP. To better assess this issue, we now compare the solution $h$ with $h_{0}$.

Figure 12 shows the profiles of $h, h_{x}$, and $h_{x x}$ for the Newton-Raphson solutions at different values of $h^{*}$, and for $h_{0}$. Note the overshoots near $x_{1}=0.229$ and $x_{2}=0.402$ in the profiles of $h_{x x}$ as $h^{*}$ goes to zero. This indicates a lack of pointwise convergence in the second derivative. We have computed the $L^{2}, H^{1}$ and $H^{2}$ norms of $h-h_{0}$ as functions of $h^{*}$ (plots not shown), and found convergence of $h$ towards $h_{0}$ as $h^{*} \rightarrow 0$, although convergence of the $H^{2}$ norm is very slow. There are several possible explanations for this fact. First of all, the solutions $h$ are estimated numerically, and convergence of the 


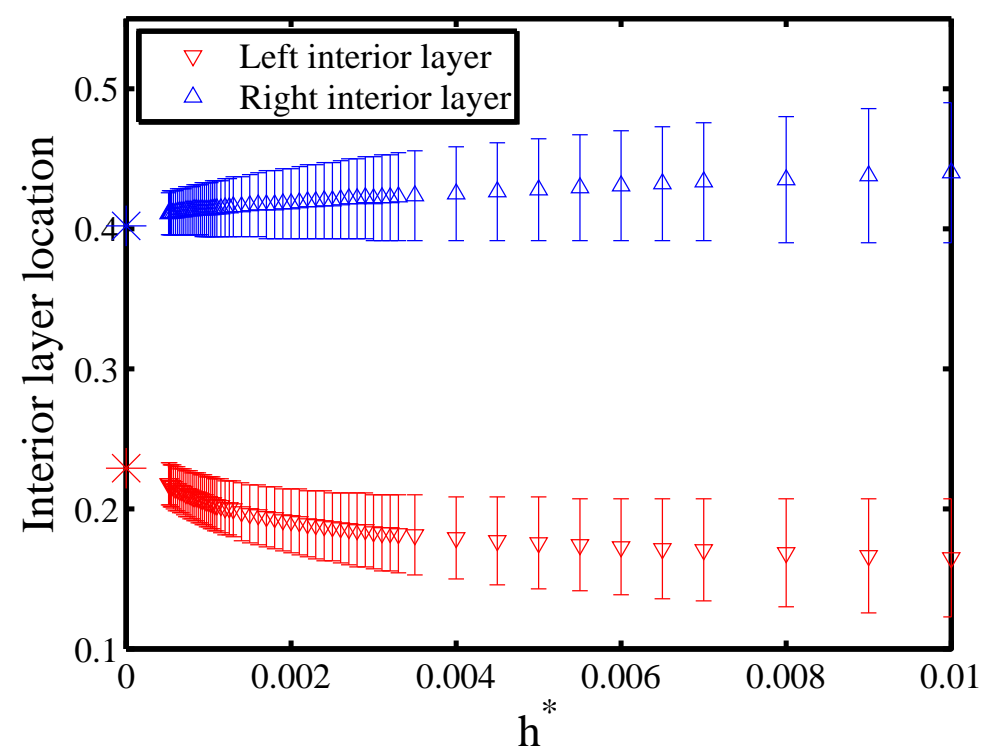

FiguRE 11. Numerically estimated values of $x_{1}$ (down triangles) and $x_{2}$ (up triangles) for the solutions obtained by Newton-Raphson iterations as $h^{*} \rightarrow 0$. The error bars indicate the width $\Delta x$ of each interior layer. The two points at $h^{*}=0$ correspond to the values $x_{1}=0.229$ and $x_{2}=0.402$. They define a solution $h_{0}$, which is one of the singular solutions that exist when DP $=0$.

Newton-Raphson iterations is very sensitive for small values of $h^{*}$. This is because $h$ gets close to zero as $h^{*}$ is decreased, and the Netwon-Raphson method may either cease to converge, or converge to a solution with negative values of $h$. Second, the asymptotic values of $x_{1}$ and $x_{2}$ are first interpolated from Figure 11, and used to identify a point on the branch of solutions that exist when $\mathrm{DP}=0$. A small error in the choice of $h_{0}$ can lead to poor decay of the norms as $h^{*} \rightarrow 0$. Third, it is possible that convergence only occurs in the weak sense. More generally, a numerical investigation can only identify trends that may inform further analysis. However, a full analytical study of the behavior 

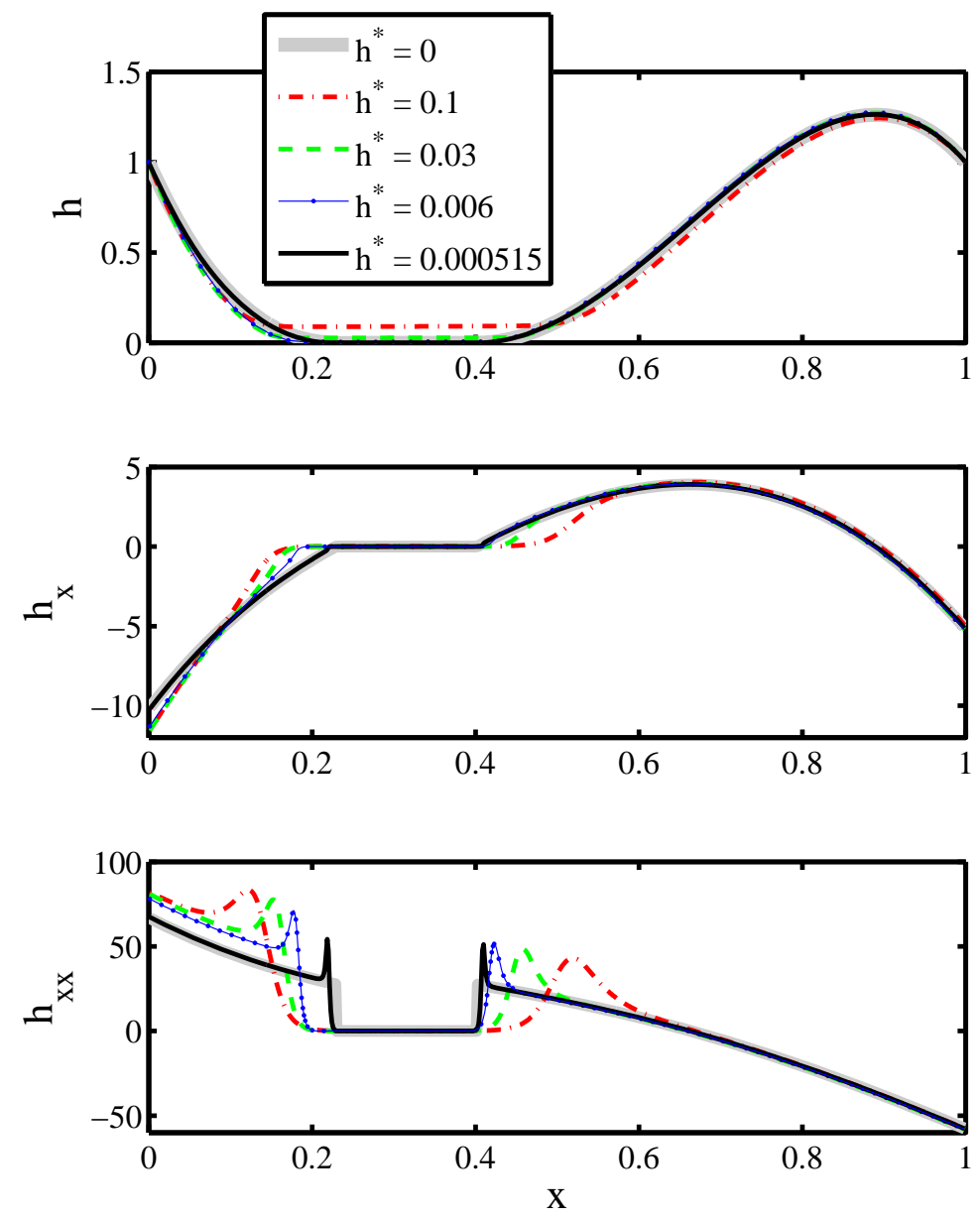

Figure 12. Solutions $h$ (top) of Equation (3.2) with (BC1) boundary conditions, their first derivatives $h_{x}$ (middle), and second derivatives $h_{x x}$ (bottom), for various values of $h^{*}$. The solution $h_{0}$ associated with DP $=0$ is shown as a thick gray curve. Parameters are $\lambda=1$, $\sigma=0.004, V=0.5, A=1, n=4, m=3$. The number of mesh points in the interval $(0,1)$ is 700.

of the solutions as $h^{*}$ goes to zero is, as mentioned above, rather impractical, and beyond the scope of this paper. 
3.4.2 As $A \rightarrow 0$

A decrease in $h^{*}$ corresponds to a smaller film thickness at which the disjoining pressure kicks in. A smaller value of $A$, on the other hand, corresponds to a decrease in the magnitude of the disjoining pressure. Here we explore convergence of the equilibrium solutions as $A \rightarrow 0$. In relation to Section 3.2 , note that the matched asymptotic expansion was performed assuming $h^{*}$ to be a small parameter and $A$ to be $O(1)$. Physically, it is always the case that $h^{*} \ll 1$, whereas $A$ is not necessarily small. Thus, the matched asymptotic analysis does not apply to the limit $A \rightarrow 0$.

In Figure 13 equilibrium profiles of $h, h_{x}$, and $h_{x x}$ are plotted for decreasing values of $A$. As before, these solutions are obtained by Newton-Raphson iterations, similar to what was done in Section 3.4.1, except that now $A$ is varied while $h^{*}$ remains equal to 0.05. The case $A=0$ corresponds to a particular solution from the continuum family that exists when DP is zero. The corresponding values of $x_{1}$ and $x_{2}$ were interpolated from Figure 14, and a closely matching point on the continuum of solutions at DP $=0$ was selected. The values of $x_{1}$ and $x_{2}$ are equal to 0.192 and 0.396 respectively, and marked in Figure 14 by stars on the $A=0$ axis. As before, the solutions with DP appear to be converging to a solution without DP in the limit of $A$ going to zero. Interestingly, the solution with $A=0$ is also an interior member of the continuum of solutions, but it is different from the one selected as $h^{*}$ goes to zero.

In Figure 13, the profiles of $h$ and $h_{x}$ when $A=10^{-5}$ are indistinguishable from the solution with $A=0$. Also, the second derivative is still quite smooth, and is approaching the discontinuous solution without overshoot. The sequence of solutions $h$ selected as 

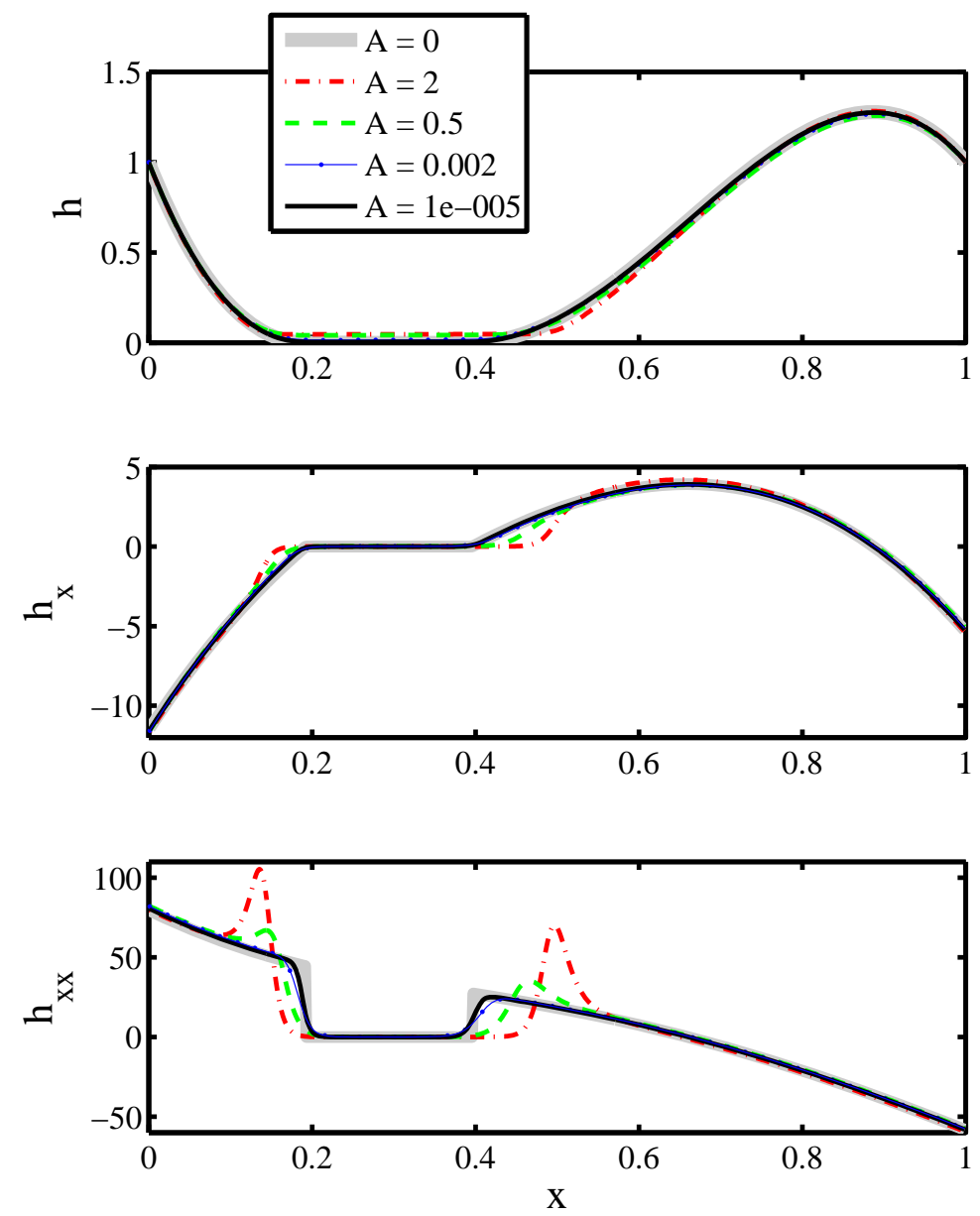

Figure 13. Solutions $h$ (top) of Equation (3.2) with (BC1) boundary conditions, their first derivatives $h_{x}$ (middle), and second derivatives $h_{x x}$ (bottom), for decreasing values of $A ; \lambda=1$, $\sigma=0.004, V=0.5, h^{*}=0.05, n=4, m=3$. The solution with $A=0$ (thick, gray curve) has its second derivative discontinuous at $x_{1}=0.192$ and $x_{2}=0.396$. The number of mesh points in the interval $(0,1)$ is 700 .

$A \rightarrow 0$ is therefore markedly different from the sequence with $h^{*} \rightarrow 0$ (compare the bottom panel of Figure 13 with that of Figure 12). However, convergence of $h_{x x}$ to $h_{0 x x}$ is very slow, and the $H^{2}$ norm of $h-h_{0}$ is still of order one at $A=10^{-5}$. 
Effect of disjoining pressure in a thin film equation with non-uniform forcing

The above experiments reveal different types of convergence as $A$ and $h^{*}$ go to zero. The two parameters play different roles since $A$ is the overall strength of the disjoining pressure term and $h^{*}$ is the value of $h$ at which $\Pi(h)$ vanishes. The limiting profiles have different points of discontinuity in their second derivatives, which were estimated to be $x_{1}=0.192$ and $x_{2}=0.396$ for $A \rightarrow 0$, and $x_{1}=0.229$ and $x_{2}=0.402$ for $h^{*} \rightarrow 0$. But the most striking feature is the behavior of the second derivatives of the solutions for small $A$ and small $h^{*}$, suggesting that $h_{x x}$ converges pointwise almost everywhere to its limiting discontinuous profile as $A \rightarrow 0$, but not necessarily as $h^{*} \rightarrow 0$. A rigorous elucidation of the nature of the convergence of equilibrium solutions as $A$ or $h^{*}$ is sent to zero is an interesting analysis question that goes beyond the scope of this paper. It would shed light on the results of the current study, which is limited by the use of numerical simulations. In particular, the Newton-Raphson method we use solves (3.2) with (BC1) with an accuracy of $10^{-8}$ at every mesh point, and solutions obtained from the full dynamic simulations of (1.3) similarly satisfy (3.2) for large simulation times. But a numerical evaluation of $Q(h, x)$ on these solutions shows variations in $Q$ (which is itself of order one) of the order of $10^{-2}$ or $10^{-3}$; even though the variability is reduced as the number of points is increased, this nevertheless highlights the limitations of the numerical approach in the presence of very large third order derivatives of $h(x, t)$, which are to be expected as $A \rightarrow 0$ or $h^{*} \rightarrow 0$.

\section{Dynamics in the presence of DP and body force, coarsening effect}

In this section, we consider how the competition between body force and DP affects the dynamics of the system. Without forcing, a flat solution is unstable to long wavelength 


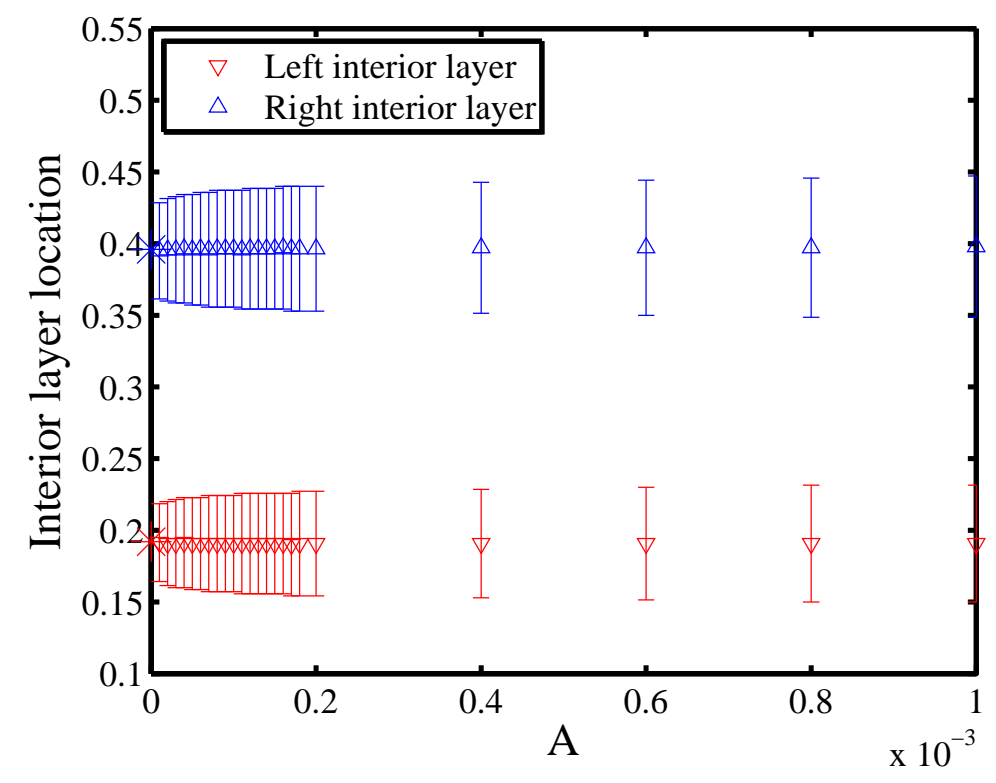

FiguRE 14. Numerically estimated values of $x_{1}$ (down triangles) and $x_{2}$ (up triangles) for the solutions obtained by Newton-Raphson iterations as $A \rightarrow 0$. The error bars indicate the width $\Delta x$ of each interior layer. The points at $A=0$ correspond to the values $x_{1}=0.192$ and $x_{2}=0.396$. They define a solution $h_{0}$, which is one of the singular solutions that exist when $\mathrm{DP}=0$.

perturbations in the presence of DP. We are interested in (i) how wavenumber selection is affected by a periodic body force whose period is not commensurate with that favored by DP, and (ii) the coarsening effect of the DP, and how this is influenced by the body force. For this section, we therefore use boundary conditions (BC2), since no coarsening is observed with boundary conditions (BC1). In the examples that follow, we implement a flat initial profile, $h(x, 0)=V$, with $V$ not necessarily equal to 0.5 as was the case until now. 


\subsection{Dynamics with and without DP}

Figure 15 shows the film profile at three different times during a simulation of (1.3), with $\gamma(x)$ given by (1.2) with $\lambda=1.9$. The left column has no DP, the right column has $A=1$ and $h^{*}=0.02$. All other parameters are equivalent and boundary conditions are (BC2). Without DP the equilibrium state consists of 2 disconnected drops ${ }^{2}$, whereas in the case with DP, all of the fluid except for a very thin layer is collected in a single drop on the right side. Dynamically, the evolution with DP is intriguing: the film comes very close to the equilibrium state without DP (compare the second picture with DP to the 4th picture without), but does not remain. The drop on the left very slowly drains through the connecting "bridge" of fluid, until eventually only the single drop remains.

The previous example suggests a coarsening effect of the DP - multiple droplets form but subsequently merge to form a single droplet. The number of droplets that initially form depends strongly on both the form of the body force and on the parameters in the DP. Regions where $\gamma>0$ represent spatial regions where the force in the positive $x$ direction is stronger while regions where $\gamma<0$ correspond to a stronger force in the negative $x$ direction. Without DP, the maximum number of "droplets" is connected to the number of roots of $\gamma(x)[20]$. The more roots $\gamma(x)$ has in the interval [0,1], the more points where the directionality of the body force reverses, and thus more droplets and more complex equilibrium states are possible. Thus, if we decrease $\lambda$ so that $\gamma(x)$ has no

2 The word "drops" evokes references to a film on a solid substrate. While we use the word for convenience, the reader should keep in mind that in the case of a soap film, the drops refer to bands of thicker film. 
Without DP
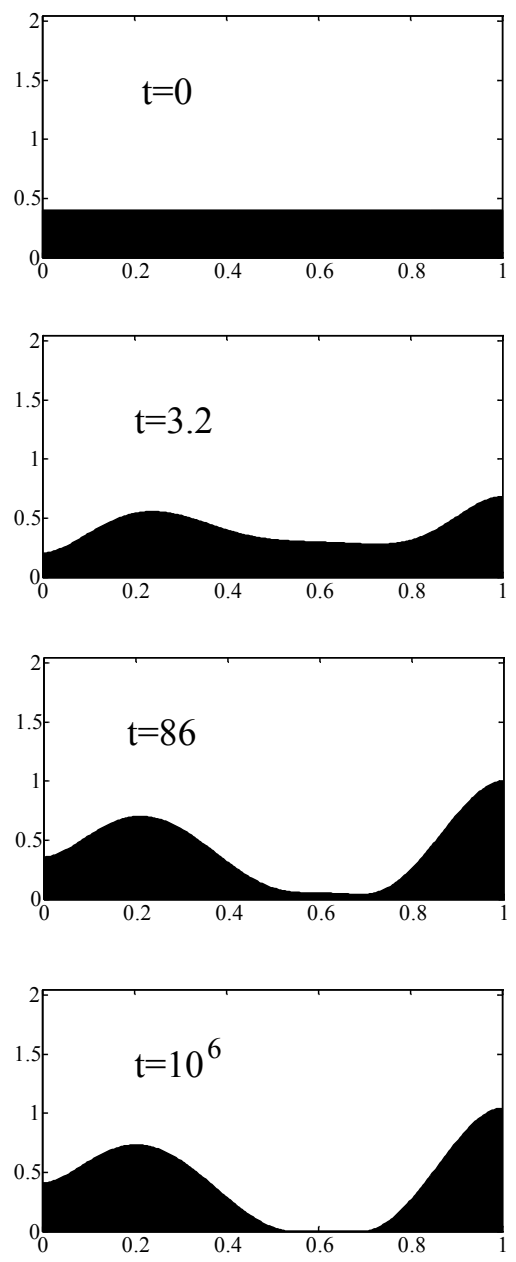

With DP
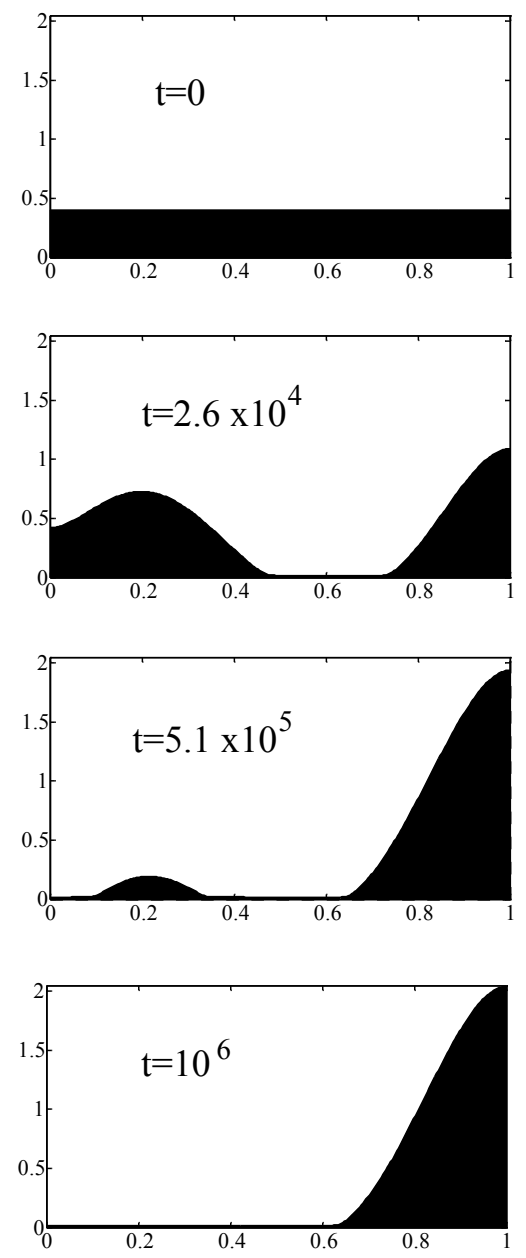

FigURE 15. Evolution starting from a flat film with and without DP; $\lambda=1.9, \sigma=0.001$,

$V=0.4$, and for the right column $A=1, h^{*}=0.02$. Boundary conditions are (BC2).

roots in $x \in[0,1]$ (i.e. if $\lambda<\lambda_{c}=8^{4} /\left(3 \cdot 7^{3} \sqrt{7}\right) \simeq 1.5045$ for the forcing $\gamma(x)$ given in (1.2)), no droplets form and all the fluid collects on the right boundary. Physically, for a small enough magnetic force, gravity dominates and drives the flow down (i.e. to the right boundary of the domain). 


\subsection{Wavelength selection, body force and coarsening}

We now consider the effect of DP and body force on the wavelength selection and pattern formation in the early dynamics. We first set $\gamma(x)=0$ to focus on the effect of DP, and note that an initially flat film $h(x, 0)=V$ is an equilibrium solution in absence of external force. Consider a perturbation from that solution of the form

$$
h(x, t)=V+\epsilon e^{i 2 k \pi x+w t} .
$$

Inserting into the evolution equation (1.3) and expanding, we obtain at $\mathrm{O}(\epsilon)$ the relation

$$
w(k)=\frac{4}{3} V^{2} \pi^{2} k^{2}\left(A\left\{m\left(\frac{h^{*}}{V}\right)^{m}-n\left(\frac{h^{*}}{V}\right)^{n}\right\}-4 \sigma V \pi^{2} k^{2}\right) .
$$

For $V$ small enough, there is a band of unstable modes (with $w(k)>0$ ) centered at $k=0$, indicating a long-wavelength instability. The most unstable mode is the one that maximises $w$, which is found to occur at $\pm k_{c r}$ with

$$
k_{c r}:=\frac{1}{4 \pi} \sqrt{\frac{2 A\left\{m\left(\frac{h^{*}}{V}\right)^{m}-n\left(\frac{h^{*}}{V}\right)^{n}\right\}}{\sigma V} .}
$$

Equation (4.3) defines the most unstable wavelength for the flat film, and in a finite domain dictates the number of "drops" formed in the early dynamics of the film evolution in the absence of body force. Note that for $V$ large enough, all modes are stable, that is $w(k)<0$ for all $|k| \geq 1$, in which case the flat film is stable. Decreasing $V$ increases the most unstable mode. Physically, this reflects the fact that a very thin film will initially form into a higher number of drops, while a thick enough film does not form into drops at all. 

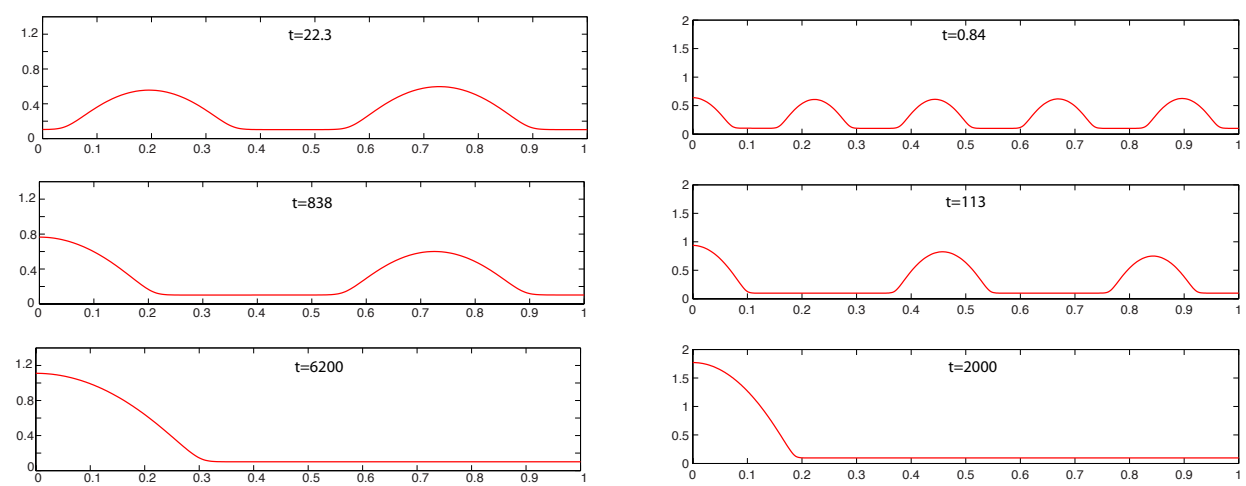

FIgURE 16. Film evolution in the absence of body force. A multiple-drop state initially forms based on the critical wavenumber, followed by coarsening. Parameters are $\sigma=0.001, h^{*}=0.1$, $V=0.3$ and (left) $A=1.5\left(\Rightarrow k_{c r} \approx 2\right)$, (right) $A=10\left(\Rightarrow k_{c r} \approx 5\right)$. Boundary conditions are (BC2).

An example of the wavelength selection is given in Figure 16. Here the initial configuration is a flat film plus a random perturbation. After forming a multiple-drop state governed by the value of $k_{c r}$, coarsening occurs. Due to the DP, a thin film with width on the order of $h^{*}$ remains between the drops, connecting them; these connecting "bridges" enable flow between the drops and merging to occur.

However, the body force can also induce a wavelength on the film. To illustrate the connection more clearly, we now consider a simple sinusoidal form for the body force,

$$
\gamma(x)=\lambda \cos (2 \hat{k} \pi x)
$$

Consider the evolution in the absence of DP, again starting from a flat film. An immediate consequence of the body force is that with $\gamma \neq 0$, the flat film is not an equilibrium state. Hence no perturbation is needed, the body force itself drives the number of droplet 
states. In Figure 17, we show the evolution for $\lambda=5$ and with $\hat{k}=2$ (a) and $\hat{k}=4$ (b). The film quickly evolves to a state of multiple drops governed by the wavenumber $\hat{k}$. With no DP, multiple-drop equilibrium states exist, in which the "bridge" of fluid thins and vanishes, that is $h \rightarrow 0$ between the drops. Thus, without DP we find that no coarsening occurs, and the final profile plotted is essentially an equilibrium profile. Observe that the initial drop formation occurs on a very short time scale, while equilibrium is only reached in the limit $t \rightarrow \infty$. Clearly, the number of roots of $\gamma$ directly correlates with the initial wavelength induced in the film and the initial number of droplets. The reader is referred to [20] for a discussion of how the functional form of $\gamma(x)$ affects the possible number of drops.

When both DP and the body force are present, there are potentially two natural wavelengths in the system: the wavelength selected by the DP and Equation (4.3), and the wavelength induced by the body force, e.g. the value of $\hat{k}$ in Equation (4.4). Thus, a competition for the profile of the initial multi-drop state can be induced. To illustrate, we set the DP parameters corresponding to a fastest growing wavenumber $k=5$. We then set $\hat{k}=2$ in Equation (4.4) and vary the strength of the body force through $\lambda$. Figure 18 shows the initial multi-drop state for $\lambda=0.1,1,5,50$. It is apparent when $\lambda=0.1$ that the DP wavelength is dominant. As $\lambda$ is increased, the initial state appears as a superposition of large drops whose number matches that dictated by the body force, and smaller drops whose number resembles that dictated by DP. For large $\lambda$, the small drops 
(a)

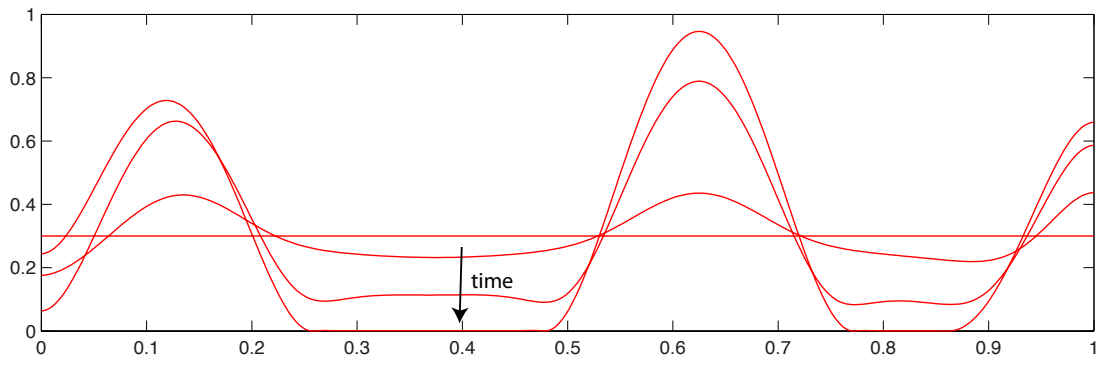

(b)

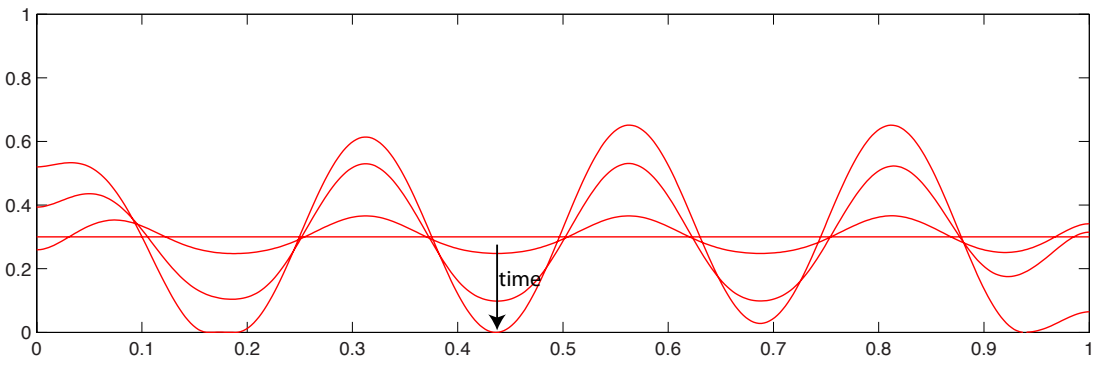

FiguRE 17. Film evolution in the absence of DP and with body force $\gamma(x)=5 \cos (2 \hat{k} \pi x)$, where $\hat{k}=2$ (a) and $\hat{k}=4$ (b). Parameters are $\sigma=0.001, V=0.3$, and curves are plotted at times (a): $t=0.18,1.8,19,300$, and (b): $t=0.05,0.4,60,000$. Boundary conditions are (BC2).

essentially vanish and the profile roughly matches the wavenumber dictated by the body force.

Also of interest is the timescale and dynamics of coarsening with both body force and DP present. In Figure 19, we show waterfall plots to illustrate the coarsening as both $\lambda$ and $A$ are varied. The effect of decreasing $A$ is generally to increase the coarsening time scale. This is intuitive, since with no DP, no coarsening is observed. If the body force is absent or negligible, the most unstable wavelength changes significantly with $A$, and so the coarsening dynamics can be quite different. The effect of $A$ on the coarsening is most 
(a)

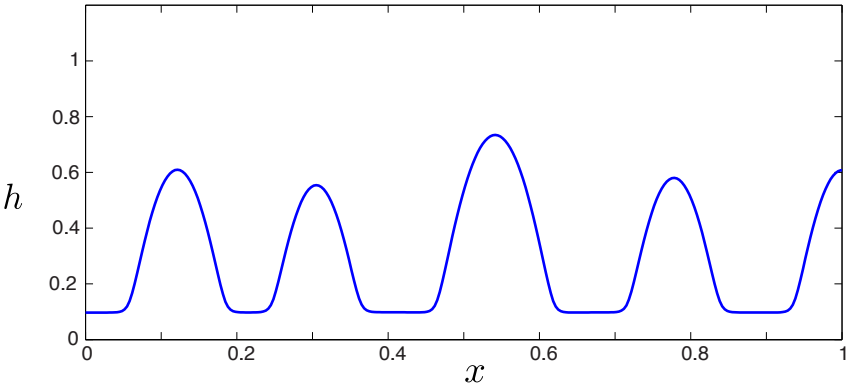

(b)

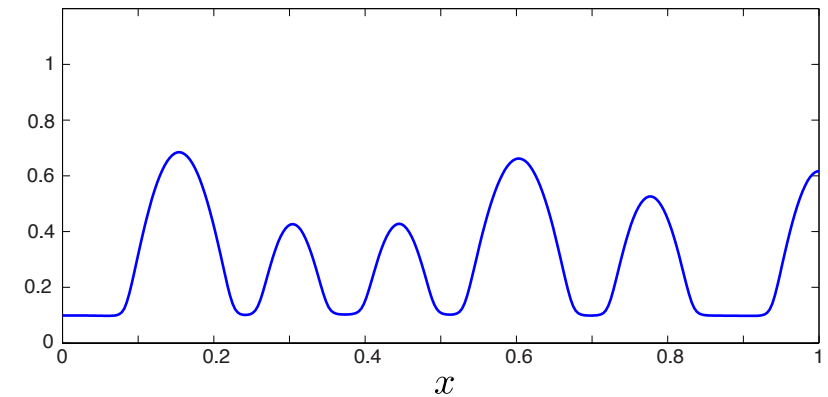

(c)

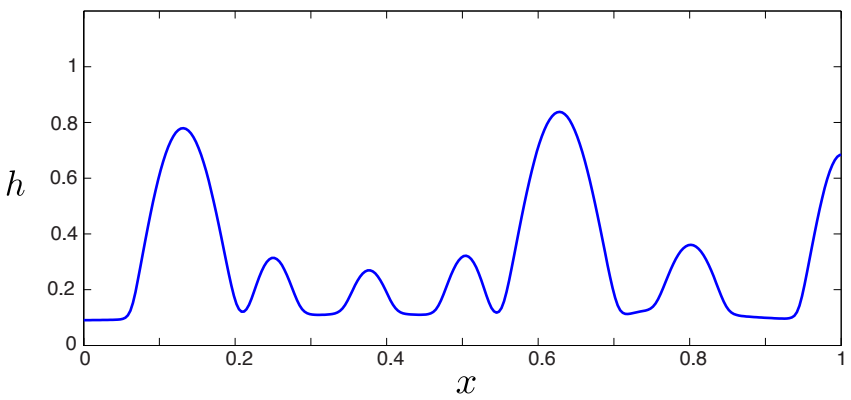

(d)

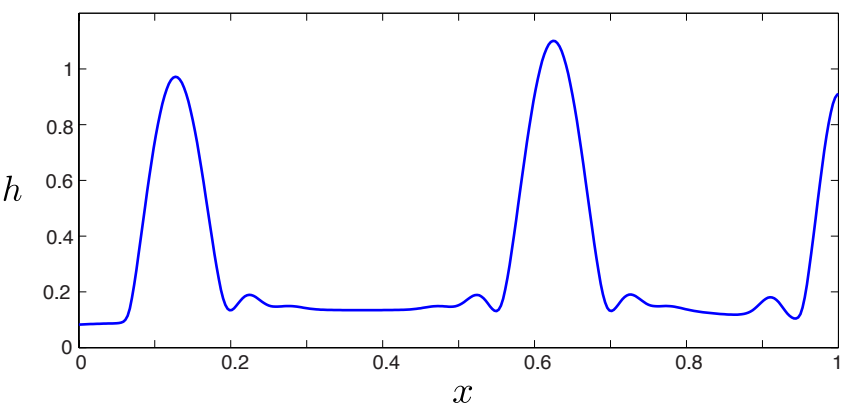

Figure 18. Initial multi-drop state. The wavenumber selected by the DP is $k=5$, while the wavenumber selected by the body force is $\hat{k}=2$. Only the initial drop state is shown, in each case full coarsening subsequently occurs. Parameters are $A=10, \sigma=0.001, h^{*}=0.1, V=0.3$, and $\gamma=\lambda \cos (4 \pi x)$ with $\lambda=0.1$ (a), 1 (b), 10 (c), and 50 (d). Boundary conditions are (BC2). 
apparent when a significant body force is also present, as seen in the right column of Figure 19. Here, 4 drops initially form for all values $A$. For large $A$, the coarsening occurs on a very short time scale; as $A$ is decreased, the time scale increases. It is to be noted that the final 4-drop profile for the upper right evolution is in fact a stable equilibrium profile; that is, at this balance of DP and body force coarsening does not occur. Thus, we see the important effect that a non-constant body force can dramatically alter the evolution such that coarsening occurs to a lesser extent or even not at all. A more comprehensive analysis of pattern selection and this transition presents an interesting study, and one we leave for future work.

\section{Conclusions}

We have investigated the role of disjoining pressure in a one-dimensional thin film evolution equation on a finite domain in the presence of a non-uniform body force with order one gradients. Our analysis has consisted in two parts. First, we investigated the structure of equilibrium solutions and the question of uniqueness in the presence of fixed height (BC1) boundary conditions. Second, we studied the effect of having both body force and DP on the wavelength selection and coarsening dynamics. For this, it was more natural to switch to fixed contact angle (BC2) boundary conditions.

Comparing to the same system without disjoining pressure, the system with disjoining pressure has a very similar structure with $\mathrm{BC} 1$, but with the key differences that the solution is regularized, film rupture does not occur, and a family of locally attracting equilibrium states is converted to a single stable equilibrium. The regularization effect of the disjoining pressure has been noted in studies without a spatially dependent body 


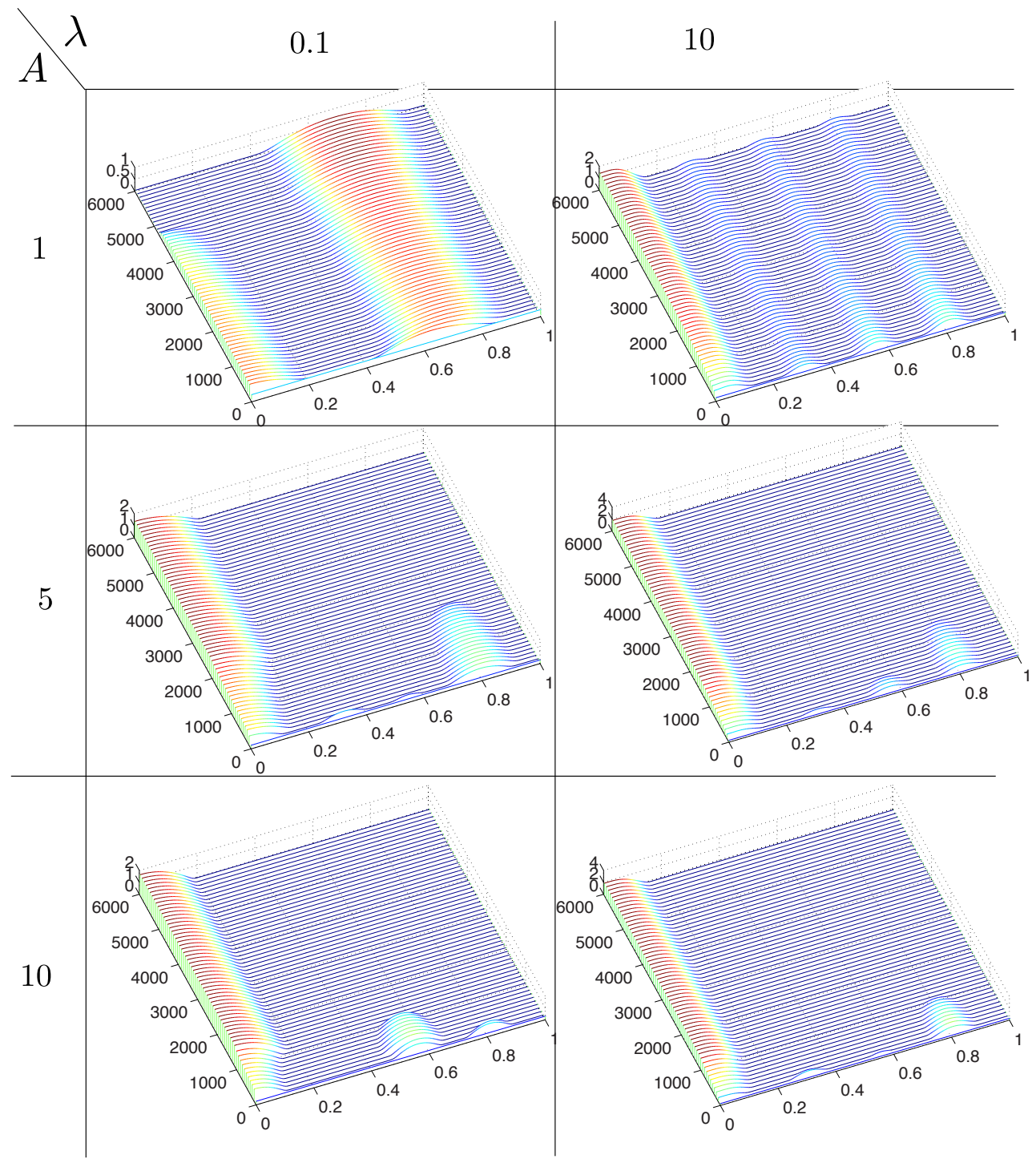

FiguRE 19. Waterfall plots to illustrate the coarsening evolution as $\lambda$ and $A$ are varied. Other parameters are $h^{*}=0.1, \sigma=0.001, V=0.3$ and $\gamma=\lambda \cos (8 \pi x)$. Boundary conditions are (BC2).

force $[12,2]$, however, the addition of the forcing function $\gamma(x)$ has several interesting and important consequences. It enables for complex solutions with an intriguing mathematical structure, which we characterized via a matched asymptotic expansion in terms 
of multiple interior layers with rapid change in the second derivative. Further, without $\gamma(x)$ the transition from a single solution with DP to multiple solutions without is not generally observed.

From a physical standpoint, we find a strong dynamical effect from the combination of DP and non-uniform forcing, due to the change from non-unique equilibrium states without DP to a unique equilibrium with DP. The existence of a continuous family of stable equilibrium solutions versus that of a single solution has a significant impact on the evolution of a film. Uniqueness means that if the equilibrium profile is globally stable, then all initial profiles of equal volume converge to the same equilibrium. As seen in Figure 10 , this seems to be the case. This is not true without DP, where initial conditions play an important role in the steady state profile [20]. Thus, for fixed height boundary conditions, a film with DP may have significantly different dynamics and equilibrium state from a film with equivalent initial profile but with DP turned off.

Moreover, in large domains or when Neumann boundary conditions are used, the DP introduces a natural wavelength into the system, which is the most unstable wavelength for a flat film. This pattern induced by the DP competes with the pattern induced in the film by the body force. In particular, the coarsening effect observed in the film evolution, which does not occur without DP, is strongly driven by $\gamma(x)$, as opposed to what was observed in previous studies where the dynamics were driven more by the initial profile [2]. Physically, in regions where $\gamma(x)>0$ there is a greater force in the negative $x$ direction, whereas $\gamma<0$ corresponds to a greater force in the positive $x$ direction. The more complex the forcing function, the more roots $\gamma$ has, the more places where the direction of the body force changes, and the more complex the fluid dynamics. In the case of zero 
Effect of disjoining pressure in a thin film equation with non-uniform forcing

contact angle (BC2), the presence of a non-uniform body force and DP has a strong effect on the details of coarsening. The coarsening dynamics is largely dependent on the initial film dynamics and the wavelength competition. With weaker DP (decreasing $A$ ), the coarsening time scale increases significantly. This has been previously noted in the absence of body force [2]. However, it is not just the timescale that is affected, but also the amount of coarsening. A film with constant body force (e.g. gravity) or no body force will undergo full coarsening, i.e. all the fluid will merge to form a single drop. This is not necessarily the case when a body force is included, as demonstrated in Figure 19. An interesting related result is found in [33], where a chemically striped substrate leads to a spatially dependent DP term. There, it was found that varying the amplitude and period of the heterogeneity creates a transition from coarsening to pinning (stable multi-drop configurations), much as varying the amplitude of a heterogeneous body force was found to do here.

The original choice for the forcing function (1.2) was motivated by the thin film study with magnetic fluids of [21], and thin film ferrofluids in the presence of non-uniform magnetic fields would seem to be the most relevant system for continued study of evolution equations with a non-uniform term $\gamma(x)$ with gradients of order one. The parameter values we have used are in rough agreement with those used in [21]. The form of $\gamma(x)$ reflects the competition between the magnetic field pulling the film upward and gravity pulling downward, and film profiles with fluid above and below a thin region were observed. Considering Figure 15, this behavior is closer to the left hand column without DP than the right hand column. However, due to evaporation and fluid leakage the film would rupture typically within a minute, and so it was not clear in those experiments 
whether the DP would have caused coarsening to occur given longer time. It would be an interesting future study to experimentally examine the coarsening effect in thin film ferrofluids, either in draining soap films or in a thin layer on a plate, and in the presence of more complex magnetic fields.

In the context of ferrofluids, to be more physically relevant, the thin film equation should be coupled to a description of the magnetic particle concentration. Further, the form of $\gamma$ in (1.2) is a simplified magnetic field which is not exact in a three-dimensional film; hence more quantitatively verifiable studies in this area will likely need a 3D model. Although the equation studied in this paper represents a simplified model, we have shown that the combination of a body force and intermolecular forces encompassed in a disjoining pressure can lead to interesting mathematical structure as well as intriguing physical film behavior.

Acknowledgements This publication is based on work supported by Award No. KUK-C1-013-04, made by King Abdullah University of Science and Technology (KAUST) (author DEM). We also thank the reviewers for their helpful comments.

\section{References}

[1] F. Bernis and A. Friedman. Higher order nonlinear degenerate parabolic equations. J. Diff. Equations, 83:179-206, 1990.

[2] A. L. Bertozzi, G. Grün, and T. P. Witelski. Dewetting films: Bifurcations and concentrations. Nonlinearity, 14:1569-1592, 2001.

[3] R. J. Braun, S. A. Snow, and S. Naire. Models for gravitationally-driven free-film drainage. J. Engineering Mathematics, 43:281-314, 2002. 
[4] R. J. Braun, S. A. Snow, and U. C. Pernisz. Gravitational drainage of a tangentiallyimmobile thick film. J. Colloid Int. Sci., 219:225-240, 1999.

[5] C.G. Broyden et al. A class of methods for solving nonlinear simultaneous equations. Mathematics of computation, 19(92):577-593, 1965.

[6] J. P. Burelbach, S. G. Bankoff, and S. H. Davis. Nonlinear stability of evaporating/condensing liquid films. J. Fluid Mech., 195:463-494, 1988.

[7] R. V. Craster and O. K. Matar. Dynamics and stability of thin liquid films. Rev. Mod. Phys., 81(3):1131-1198, 2009.

[8] P. G. de Gennes. Wetting: statics and dynamics. Rev. Mod. Phys., 57:827-880, 1985.

[9] D. A. Edwards, H. Brenner, and D. T. Wasan. Interfacial Transport Processes and Rheology. 1991.

[10] F. Elias, J.-C. Bacri, C. Flament, E. Janiaud, D Talbot, W Drenckhan, S Hutzler, and D Weaire. Magnetic soap films and magnetic soap foams. Colloids and Surf A: Physicochem. Eng. Aspects, 263:65-75, 2005.

[11] K. B. Glasner and T. P. Witelski. Collision versus collapse of droplets in coarsening of dewetting thin films. Physica D, 209:80-104, 2005.

[12] G. Grün and M. Rumpf. Simulation of singularities and instabilities arising in thin film flow. Eur. J. Appl. Math., 12:293-320, 2001.

[13] R. P. Haskett, T. P. Witelski, and J. Sur. Localized marangoni forcing in driven thin films. Physica D, 209:117-134, 2005.

[14] J. N. Israelachvili. Intermolecular and Surface Forces (second ed.). Academic Press, New York, 1992.

[15] T.B. Jones. Electromechanics of Particles. Cambridge University Press: New York, 1995.

[16] S. Kalliadasis, C. Bielarz, and G. M. Homsy. Steady free-surface thin film flows over topography. Phys. Fluids, 12(8):1889-1898, 2000.

[17] S. Kalliadasys, A. Kiyashko, and E. A. Demekhin. Marangoni instability of a thin liquid film heated from below by a local heat source. J. Fluid Mech., 475:377-408, 2003. 
[18] K. Kargupta, R. Konnur, and A. Sharma. Instability and pattern formation in thin liquid films on chemically heterogeneous substrates. Langmuir, 16:10243-10253, 2000.

[19] V. S. Mitlin. Dewetting of solid surface: Analogy with spinodal decomposition. J. Colloid Int. Sci., 156:491-497, 1993.

[20] D. E. Moulton and J. Lega. Reverse draining of a magnetic soap film - analysis and simulation of a thin film equation with non-uniform forcing. Physica D, 238:2153-2165, 2009.

[21] D. E. Moulton and J. A. Pelesko. Reverse draining of a magnetic soap film. Phys. Rev. E, 81:046320, 2010 .

[22] A. Oron and S. G. Bankoff. Dynamics of a condensing liquid film under conjoining/disjoining pressures. Phys. Fluids, 13:1107-1117, 2001.

[23] A. Oron, S. H. Davis, and S. G. Bankoff. Long-scale evolution of thin liquid films. Rev. Modern Phys., 69(3):931-980, 1997.

[24] A. Oron and P. Rosenau. Formation of patterns induced by thermocapillarity and gravity. J. Phys. II France, 2:131-146, 1992.

[25] L. M. Pismen and U. Thiele. Asymptotic theory for a moving droplet driven by a wettability gradient. Phys. Fluids, 18:042104, 2006.

[26] N. Savva and S. Kalliadasis. Two-dimensional droplet spreading over topographical substrates. Phys. Fluids, 21(9):092102, 2009.

[27] B. Scheid, A. Oron, P. Colinet, U. Thiele, and J. C. Legros. Nonlinear evolution of nonuniformly heated falling liquid films. Physics of Fluids, 14:4130-4151, 2002.

[28] L. W. Schwartz and R. R. Eley. Simulation of droplet motion on low-energy and heterogeneous surfaces. J. Colloid Int. Sci., 202:173-188, 1998.

[29] L. W. Schwartz and R. V. Roy. Modeling draining flow in mobile and immobile films. J. Colloid Int. Sci., 218:309-323, 1999.

[30] J. M. Skotheim, U. Thiele, and B. Scheid. On the instability of a falling film due to localized heating. J. Fluid Mech., 475:1-19, 2003. 
[31] G. F. Teletzke, H. T. Davis, and L. E. Scriven. How liquids spread on solids. Chem. Eng. Comm., 155:41-81, 1987.

[32] U. Thiele. On the depinning of a drop of partially wetting liquid on a rotating cylinder. $J$. Fluid Mech., 671:121-136, 2011.

[33] U. Thiele, L. Brusch, M. Bestehorn, and M. Bär. Modelling thin-film dewetting on structured substrates and templates: Bifurcation analysis and numerical simulations. Eur. Phys. J. E, 11:255-271, 2003.

[34] D. Tseluiko, M. G. Blyth, D. T. Papageorgiou, , and J.-M. Vanden-Broeck. Viscous electrified film flow over step topography. SIAM J. Appl. Math., 70:845-865, 2009.

[35] D. Tseluiko and D. T. Papageorgiou. Wave evolution on electrified falling films. J. Fluid Mech., 556:361-386, 2006.

[36] T. P. Witelski and A. J. Bernoff. Stability of self-similar solutions for van der waals driven thin film rupture. Phys. Fluids, 11:2443-2445, 1999.

[37] Y. Zhang. Counting the stationary states and the convergence to equilibrium for the 1-d thin film equation. Nonlin. Analysis, 71:1425-1437, 2009. 



\section{RECENT REPORTS}

12/54 The Stokes boundary layer for a thixotropic or antithixotropic fluid

McArdle

Pritchard

Wilson

12/55 Thermoviscous Coating and Rimming Flow

Leslie

Wilson

Duffy

$12 / 56$ On the anomalous dynamics of capillary rise in porous media

Shikhmurzaev Sprittles

12/57 Compactly supported radial basis functions: how and why?

Zhu

12/58 Multiscale reaction-diffusion algorithms: pde-assisted Brownian dynamics

Franz

Flegg

Chapman

Erban

12/59 Numerical simulation of shear and the Poynting effects by the fi-

Mihai nite element method: An application of the generalised empirical inequalities in non-linear elasticity

Goriely

12/60 From Brownian dynamics to Markov chain: an ion channel example

Chen

Erban

Chapman

12/61 Three-dimensional coating and rimming flow: a ring of fluid on a rotating horizontal cylinder

Leslie

Wilson

Duffy

12/62 A two-pressure model for slightly compressible single phase flow in bi-structured porous media

Soulaine

Davit

Quintard

12/63 Mathematical modelling plant signalling networks

Muraro

Byrne

King

Bennett

12/64 A model for one-dimensional morphoelasticity and its application to fibroblast-populated collagen lattices

Menon

Hall

McCue

McElwain

12/65 Effective order strong stability preserving RungeKutta methods

Hadjimichael

Macdonald

Ketcheson

Verner

12/66 Morphoelastic Rods Part I: A Single Growing Elastic Rod

Moulton

Lessinnes

Goriely

12/67 Wrinkling in the deflation of elastic bubbles

Aumaitre

Knoche

Cicuta

Vella 
12/70 An estimate of energy dissipation due to soil-moisture hysteresis McNamara

12/71 The Mathematics Behind Sherlock Holmes: A Game of Shadows Goriely

Moulton

12/72 Some observations on weighted GMRES Güttel

Pestana

12/73 Bounds on the solution of a Cauchy-type problem involving a Furati weighted sequential fractional derivative

12/74 Static and dynamic stability results for a class of three- Majumdar dimensional configurations of Kirchhoff elastic rods Goriely

12/75 Error estimation and adaptivity for incompressible, nonlinear (hy- Whiteley per)elasticity Tavener

12/76 A note on heat and mass transfer from a sphere in Stokes flow at Bell low Péclet number Byrne

Whiteley

Waters

Copies of these, and any other OCCAM reports can be obtained from:

Oxford Centre for Collaborative Applied Mathematics

Mathematical Institute

24 - 29 St Giles'

Oxford

OX1 3LB

England

www.maths.ox.ac.uk/occam 